\title{
Antileukotrienes for the prevention and treatment of chronic lung disease in very preterm newborns: a systematic review
}

\author{
Marlide Jukema ${ }^{1}$, Franciszek Borys ${ }^{2}$, Greta Sibrecht ${ }^{3}$, Karsten Juhl Jørgensen ${ }^{4}$ and Matteo Bruschettini ${ }^{5^{*}}$ (0)
}

\begin{abstract}
Background: Very preterm infants are at high risk of developing chronic lung disease, which requires respiratory support and might have long-term sequelae. As lung inflammation plays an important role in pathogenesis, antileukotrienes have been explored in both clinical and animal studies. We aimed to assess the benefits and harms of antileukotrienes for the prevention and treatment of respiratory morbidity and mortality in very preterm newborns.

Methods: In this systematic review, we included randomized trials and non-randomized studies in humans and animals reporting the effects of antileukotrienes in very preterm infants or other mammals within 10 days of birth. Our pre-specified primary outcomes were all-cause mortality and any harm, and, for the clinical studies, incidence of chronic lung disease. Included studies underwent risk of bias-assessment and data extraction performed by two authors independently. There were no language restrictions.
\end{abstract}

Results: Fifteen studies totally met our inclusion criteria: one randomized trial and four non-randomized studies in humans and 10 animal studies (five in rodents, two in lambs and one in either guinea pigs, rabbits or caprinae). All five clinical studies used montelukast and had a small sample size, ranging from 4 to 77 infants. The randomized trial ( $n=60)$ found no difference in the incidence of chronic lung disease between the groups. Only one clinical study, which enrolled four very preterm infants and had a critical overall risk of bias, reported long-term outcomes. All other studies had unclear or greater overall risk of bias and meta-analyses were therefore deemed unfeasible. Eight of ten animal studies used leukotriene receptor antagonists as antileukotriene (montelukast in three of ten studies) and seven had an experimental study design (i.e. some animals were not exposed to antileukotrienes but no randomization). Three of the ten animal studies assessed different doses. Animal studies found no effect on the outcomes mortality, growth, or lung function related surrogate outcomes.

Conclusions: Use of antileukotrienes in very preterm infants to prevent or treat chronic lung disease is not supported by the available evidence. Large randomized trials focusing on outcomes relevant to patients, including longterm outcomes, are needed. Studies should also minimize risk of bias.

Keywords: Preterm infants, Chronic lung disease, Animal model, Respiratory morbidity, SYRCLE

*Correspondence: matteo.bruschettini@med.lu.se

${ }^{5}$ Cochrane Sweden, Dept. Research and Development, Skåne University Hospital, Clinical Science Lund, Lund University, Lund, Sweden

Full list of author information is available at the end of the article

\section{Background}

Very preterm infants (born before 32 weeks' gestational age) constitute an extremely vulnerable population and are at high risk of developing chronic lung disease [1]. Chronic lung disease is a broad term, which includes bronchial asthma and bronchopulmonary dysplasia (BPD). It has been reported that BPD is the most 
common complication in extremely preterm infants [2]. Defining BPD remains a challenge [3]. This is mainly due to there being multiple factors involved in the underlying pathophysiology. Injury to the lungs, both before and after birth, may lead to an abnormal reparative response. This could cause flawed lung development, which can affect lung function into adult life [2]. Caffeine is the only drug that reduces the rate of BPD [4], mortality, and neurodevelopmental disability [5]. More interventions are therefore needed to prevent and treat BPD and its consequences.

Antileukotrienes include leukotriene receptor antagonists (e.g. montelukast, zafirlukast and pranlukast) and leukotriene synthesis inhibitors (e.g. zileuton) [6]. Antileukotriene receptor antagonists (LTR As) bind competitively to cysteinyl leukotriene receptors 1 and block the contractile promoting activity of leukotrienes in airway smooth muscles.

Montelukast is the most common type in clinical use, is administered once a day, and can be taken without regard to meals [7]. Zafirlukast and pranlukast are administered twice a day. The LTRA $s$ are processed mainly in the liver [8], metabolized mostly by CYP2C8, with the involvement of CYP2C9 CYP3A4 enzymes [9, 10]. It is worth mentioning that LTRAs are substrates for transporters [11] and the s of genes In children, common montelukast induced adverse events are headaches, abdominal pain, rash, thirst, hyperkinesia, asthma and eczema [13]. Pharmacovigilance studies have also reported increased frequency sleeping disorders in infants younger than 2 years and psychiatric disorders in children aged 2 to 11 years, being more frequently reported than in adults. This led to a US FDA alert being issued for psychiatric events being associated with montelukast. Eosinophilic granulomatosis may also be associated with the use of montelukast, but the role of LTRAs in its pathogenesis is still uncertain [15].

The drug zileuton, also an antileukotriene, has a different action mechanism from LTRAs. It works as an inhibitor of 5-lipoxygenase. The most serious concern is hepatotoxicity. Zileuton is mainly metabolized through the liver, particularly via P450 enzymes, mostly by CYP3A4 [16]. This can lead to problems when using drugs such as theophylline at the same time due to impaired metabolization of theophylline. An option is to halve the dose of theophylline when starting treatment with zileuton [8]. Theophylline is an example of a methylxanthine, which are known to have a protective effect on the respiratory system [17]. Methylxanthines are natural components of cocoa-based products and beverages such as coffee, tea and yerba mate and therefore are commonly present in the human milk, thus reaching the newborn.
The properties of antileukotrienes might have the potential to be useful in the prevention and treatment of chronic lung disease in very preterm infants and they are currently used clinically based on anecdotal evidence, though not approved for this purpose. Their harms and benefits have not been assessed systematically. This systematic review aims to explore the evidence base for antileukotrienes in very preterm neonates in both clinical and animal studies.

\section{Methods}

Our methods for systematically reviewing the clinical studies are based on the template developed by the Cochrane Neonatal Review group (Resources for Review Authors, n.d.) [18]. Two separate protocols were registered in Prospero for the clinical and animal studies, respectively $[19,20]$, since Prospero requires authors to register separate protocols for clinical and animal studies. An exploratory pilot search for animal studies was performed before submitting the protocols and our comprehensive search and data extraction.

\section{Types of studies}

We included randomized and non-randomized animal studies. Studies with a cross-over design were excluded due to our interest in long-term outcomes and the potential for carry-over effects.

For the clinical studies, we included randomized trials, quasi-randomized trials and non-randomized studies of intervention (NRSI). Again, we excluded trials with a cross-over design.

\section{Types of participants}

We included studies in any neonatal mammals, both term and pre term. "Neonatal" was defined as the first 10 days since birth, which is an arbitrary cut-off point that we pre-specified in our protocol. For the clinical studies, we included very preterm infants with a gestational age below 32 weeks and who were admitted to a neonatal department.

\section{Types of interventions}

For animal studies, we included studies using co-interventions and any route and dose of antileukotriene administration. We excluded studies where antileukotrienes were administered to mothers before birth or to lactating mothers. We also excluded studies where cointerventions were not available for all study arms. We included two types of studies (1) antileukotrienes versus control (either placebo, no intervention, or treatment as usual); (2) studies without any comparator (non-controlled studies). 
For the clinical studies, we included two comparisons, i.e. (1) prevention and (2) treatment of chronic lung disease.

\section{Outcomes}

\section{Animal studies}

Our primary outcomes for the animal studies were: (1) survival until last follow up; (2) any harm.

Our secondary outcomes were: (1) growth; (2) lung volume to body weight ratio; (3) lung function; (4) lung histology; (5) inflammation markers for lungs: levels of interleukins (IL), i.e. IL-1 $\beta$, IL-6, IL-16, IL-8/CXCL-8, IL10, IL-4, IL-13, CC Chemokines (MCP-1, $1 \alpha, 1 \beta, 2$ and $3)$, Krebs von den Lungen (KL-6), Clara cell secretory protein (CC16), neutrophil gelatinase-associated lipocalin (NGAL), placental growth factor, N-terminal proBNP (NT-pro-BNP), macrophage migration inhibitory factor, NF- $\kappa \beta$, Soluble ICAM, Tumor Necrosis Factor- $\alpha$, cysteinyl leukotriene (cysLT) release in bronchoalveolar lavage fluid, airway eosinophilia, mucus hyperproduction; (6) lung injury; (7) a irway hyperresponsiveness, fibrosis and smooth muscle actin expression; (8) behavioral tests; (9) markers for apoptosis; (10) pulmonary vascular resistance, Fulton index, and arterial wall structure. We included animal studies regardless of outcomes. Most of these are surrogate outcome measures, which however might provide a useful insight on pathophysiology in exploratory animal studies.

\section{Clinical studies}

Our primary outcomes for the clinical studies were: (1) all-cause mortality during initial hospitalization; (2) $\mathrm{BPD} /$ chronic lung disease incidence: only for comparison one (i.e. prevention of chronic lung disease) according to the three definitions: [21-23]; (3) any harm.

Secondary outcomes were: (1) all-cause neonatal (first 28 days) mortality, only for comparison one (i.e. prevention of chronic lung disease); (2) retinopathy of prematurity (any and $\geq$ stage 3 [24]) (3) days of respiratory support; (4) days of supplemental oxygen; (5) need for mechanical ventilation (yes/no); (6) days of hospital stay; (7) major neurodevelopmental disability: cerebral palsy, developmental delay [25, 26] or Griffiths Mental Development Scale [27] assessment greater than two standard deviations (SDs) below the mean), intellectual impairment (intelligence quotient (IQ) greater than two SDs below the mean), blindness (vision less than $6 / 60$ in both eyes), or sensorineural deafness requiring amplification. We pre-planned to assess data for children aged 18 to 24 months and aged three to five years separately; (8) each component of the composite outcome "major neurodevelopmental disability"; (9) pulmonary function test at school age (as specified by study authors).

\section{Searches}

We searched the Cochrane Central Register of Controlled Trials (CENTRAL) in The Cochrane Library; MEDLINE via PubMed, and Embase, in September 2020. We also searched ongoing clinical trials submitted at clinicaltrials.gov and ITCRP website. We did not apply any restrictions regarding language, publication year, or publication status. Methodological filters excluding diagnostic studies were not used. Search strings for each database are listed in Appendix.

\section{Selection of studies}

Two authors independently screened titles and abstracts and retrieved the full text of potentially relevant articles. Eligibility was assessed according to our inclusion criteria. Two authors independently performed data extraction and assessed risk of bias.

\section{Assessment of risk of bias}

We used SYRCLE's risk of bias tool [28] for animal studies, which include the following seven domains: selection bias due to sequence generation, baseline characteristics or inadequate allocation concealment; performance bias due to inadequate randomization housing or blinding; detection bias due to inadequate randomization of outcome assessment or blinding; attrition bias due to incomplete outcome data; reporting bias due to selective outcome reporting; and other sources of bias.

For non-randomized clinical studies, we used the ROBINS-I [29] tool to assess the risk of bias, which include the following eight domains: bias due to confounding; bias in selection of participants into the study; bias in classification of interventions; bias due to deviations from intended interventions; bias due to missing data; bias in measurement of outcomes; bias in the reported results; and the overall risk of bias. For the domain "confounding", we took into account the following confounders: antenatal steroids, gestational age, birth weight, Apgar score, indication to start antileukotrienes and level of respiratory support at study entry.

For randomized trials, we used the Cochrane Risk of Bias 2 tool [30], which include the following five domains: bias arising from the randomization process; bias due to deviations of intended interventions; bias due to missing outcome data, bias in measurements of 
the outcome; bias in selection of the reported results and overall risk of bias.

Any disagreements were solved through discussion and, if necessary, by consulting a third review author.

\section{Data analysis}

We planned to use the Cochrane software RevMan 5.4 [31] to synthesize and analyze data. We planned to analyze all infants and animals on an intention-to-treat basis and to use the fixed-effect model for meta-analyses because we expected a consistent treatment effect. We planned to synthesize data with risk ratios (RR) for dichotomous outcomes and mean differences (MD) for continuous outcomes, with $95 \%$ confidence intervals (CI). The overall certainty of the evidence was assessed using the Grading of Recommendations Assessment, Development and Evaluation (GRADE) approach, as outlined in the GRADE Handbook [32], for our primary outcomes.

\section{Subgroup analyses}

For the animal studies, we planned the following: type of lung injury, dose and type of antileukotrienes.

For the clinical studies, we planned the following: (1) gestational age: extremely preterm infants $(<28$ weeks' gestation, very preterm infants ( 28 to $31+6$ weeks' gestation; (2) type of antileukotrienes: leukotriene receptor antagonists, leukotriene synthesis inhibitors; (3) age when first dose of leukotriene receptor antagonist was given; and (4) route of administration.

\section{Results}

\section{Results of the search}

Our searches for animal and clinical studies (Appendix) returned 1929 unique records. One additional study was identified through other sources (online search) while completing the review. Following screening titles and abstract, 22 studies were collected and assessed in fulltext. Three animal studies were excluded because the animals were older than 10 days. Three studies were labelled as awaiting classification because the text of the conference abstracts were not available [33, 34] or because a protocol registered in 2007 was apparently not followed by a publication. One ongoing uncontrolled clinical study was identified, with a planned sample size of 200 very low birth weight newborns [35]. Thus, fifteen studies were included: ten animal studies (see Table 1) and five clinical studies of which one was a randomized trial (see Table 2). Figure 1 presents the PRISMA flow chart.

\section{Included studies}

Animal studies

Of the ten included animal studies, five were in rodents (three in rats and two in mice) [36-40] and two were in lambs $[41,42]$. The remaining three were in either guinea pigs [43], rabbits [44] or caprinae (sheep and goats in the same study) [45]. Two studies assessed prevention of respiratory morbidity only [36, 44], while three studied treatment effects only [37, 39, 40]. Five studied both prevention and treatment effects [38, 41$43,45]$. Eight of ten studies used leukotriene receptor antagonists such as antileukotrienes (montelukast used in three studies $[36,37,39]$ ), one studied a leukotriene synthesis inhibitor [40] and one studied both types of antileukotrienes [45]. Of the 10 animal studies, seven had an experimental study design (i.e. some animals were not exposed to antileukotrienes but were not randomized) and three an observational study design (i.e. all animals were exposed to antileukotrienes). Within three of the ten studies different doses were assessed $[40,43,44]$.

\section{Clinical studies}

The five clinical studies included one randomized trial from Korea [46], a non-randomized study performed in Germany and the USA [47], and tree observational studies from Korea [48], the UK [49] and Taiwan [50]. Four studied treatment and one studied prevention of BPD [46]. All five assessed the same leukotriene antagonist; montelukast. The administered dose of montelukast ranged from 1 to $2 \mathrm{mg} / \mathrm{kg}$ body weight. Details are provided in Table 2.

\section{Risk of bias \\ Animal studies}

Details of our risk of bias assessments are presented in Table 3. Overall, risk of bias was difficult to assess due to poor reporting and most domains were therefore "unclear" using the SYRCLE risk of bias tool [28]. As this tool is developed specifically for experimental animal studies, some domains were not applicable to the three non-controlled studies $[41,42,45]$.

The seven experimental animal studies all had unclear risk of selection bias because the randomization process and baseline characteristics were not specified. They all had an unclear risk of performance bias because none of the studies reported on random housing. The measures 


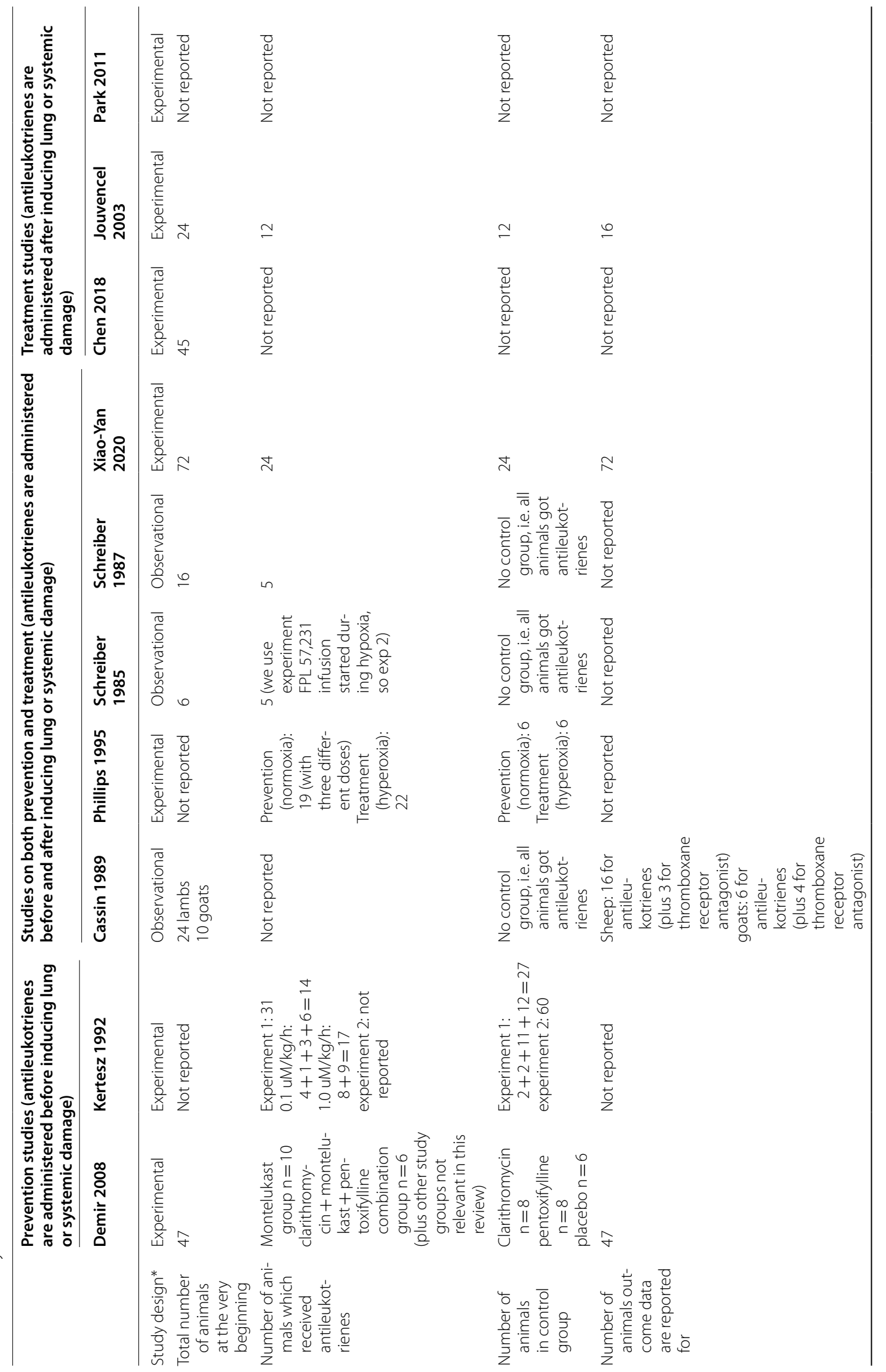




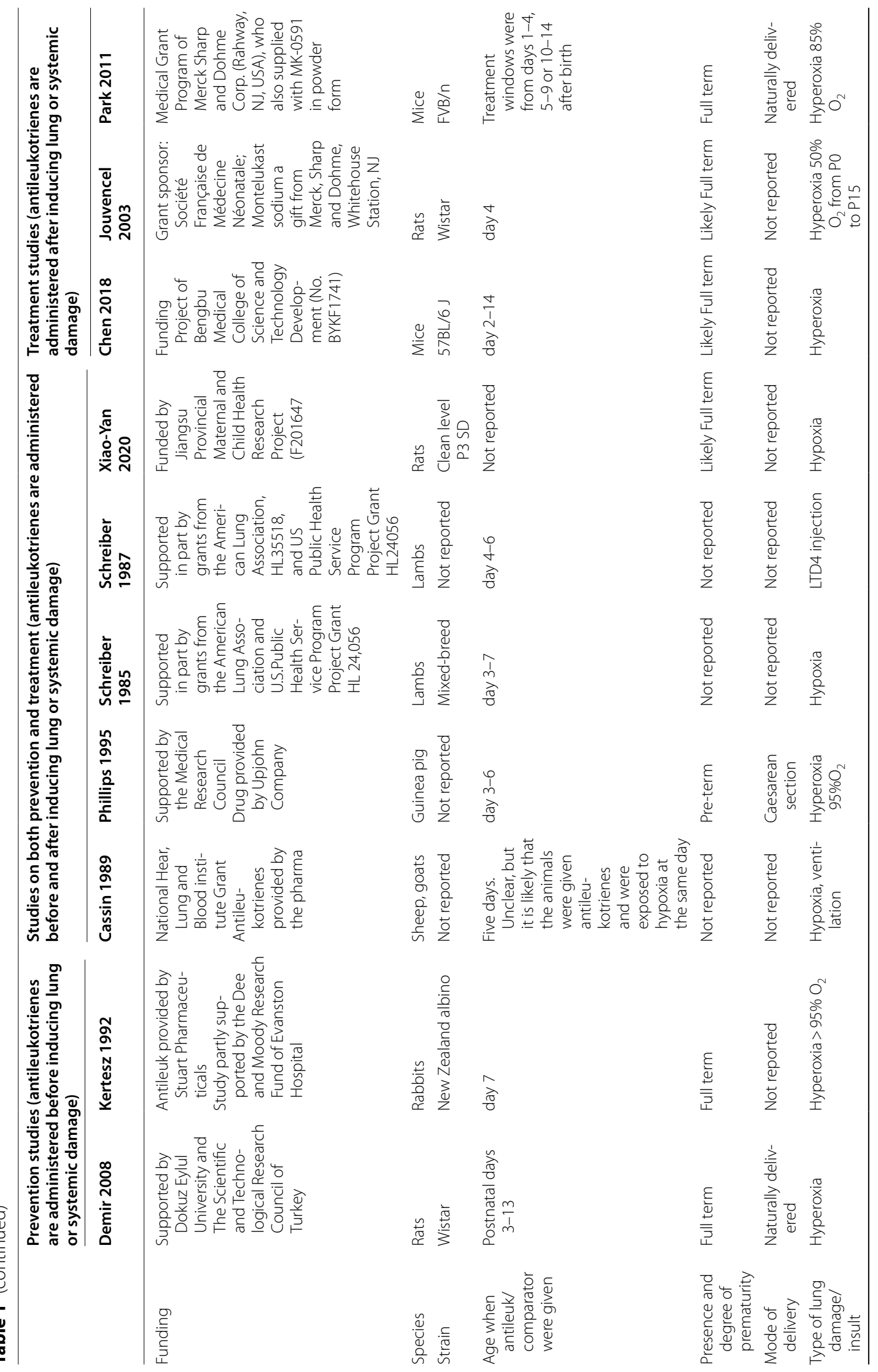




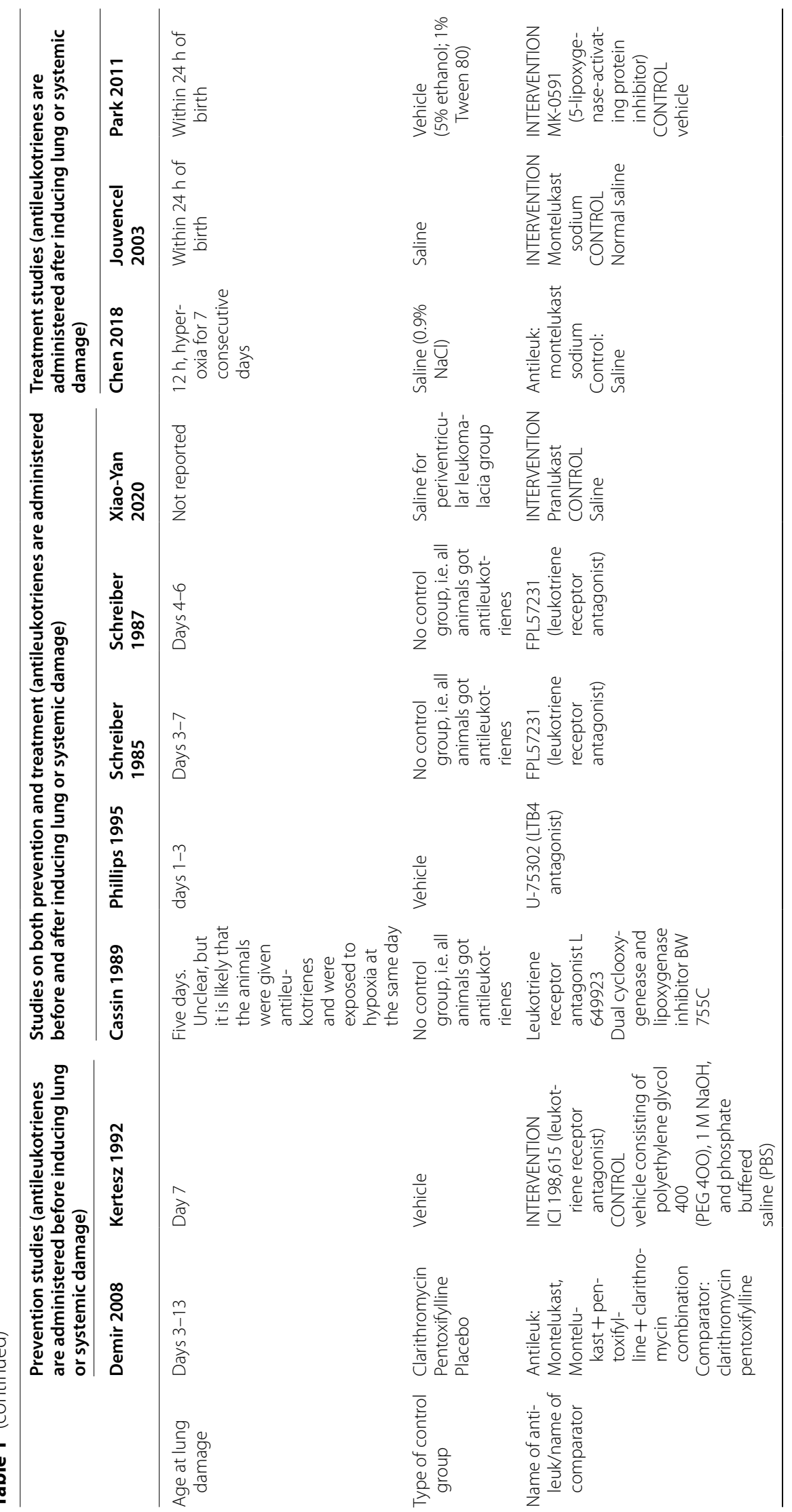




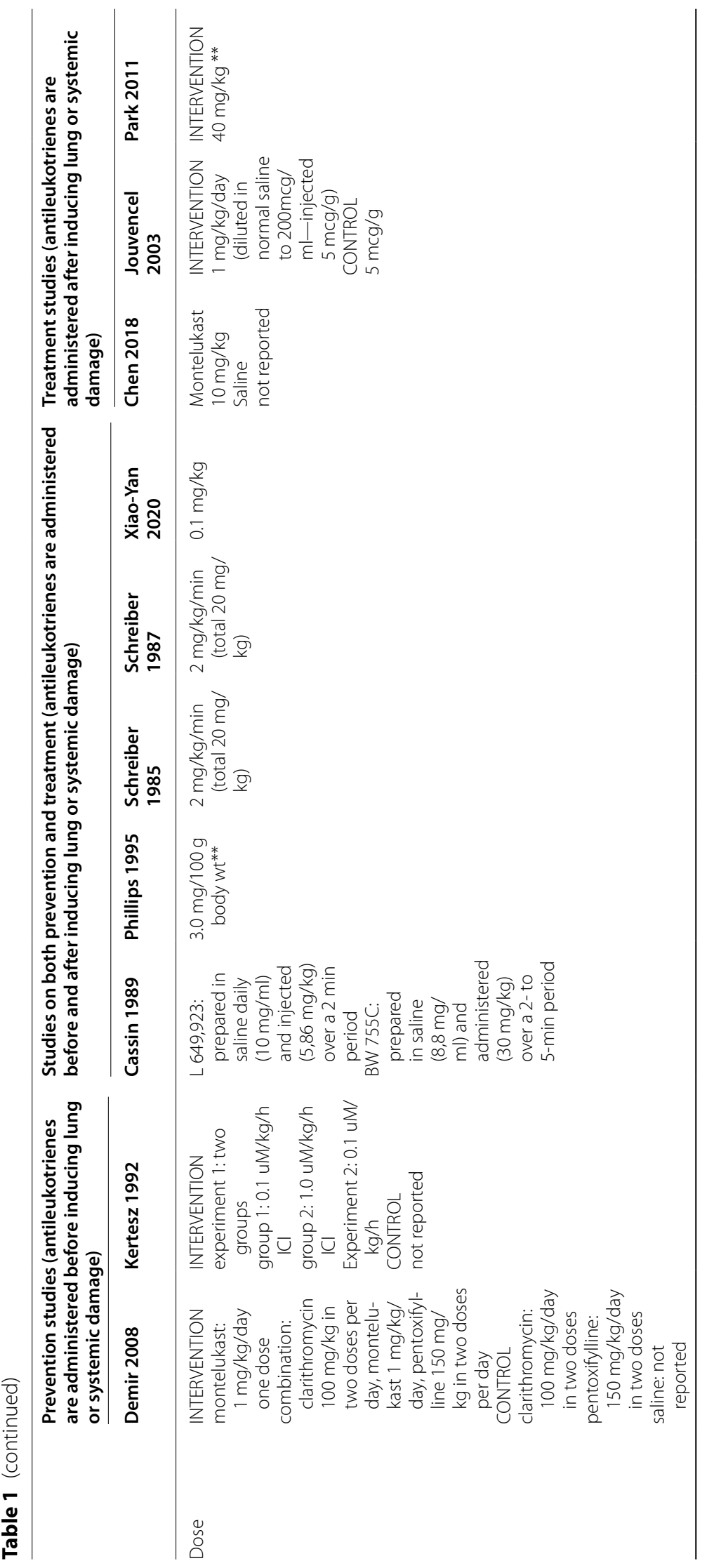




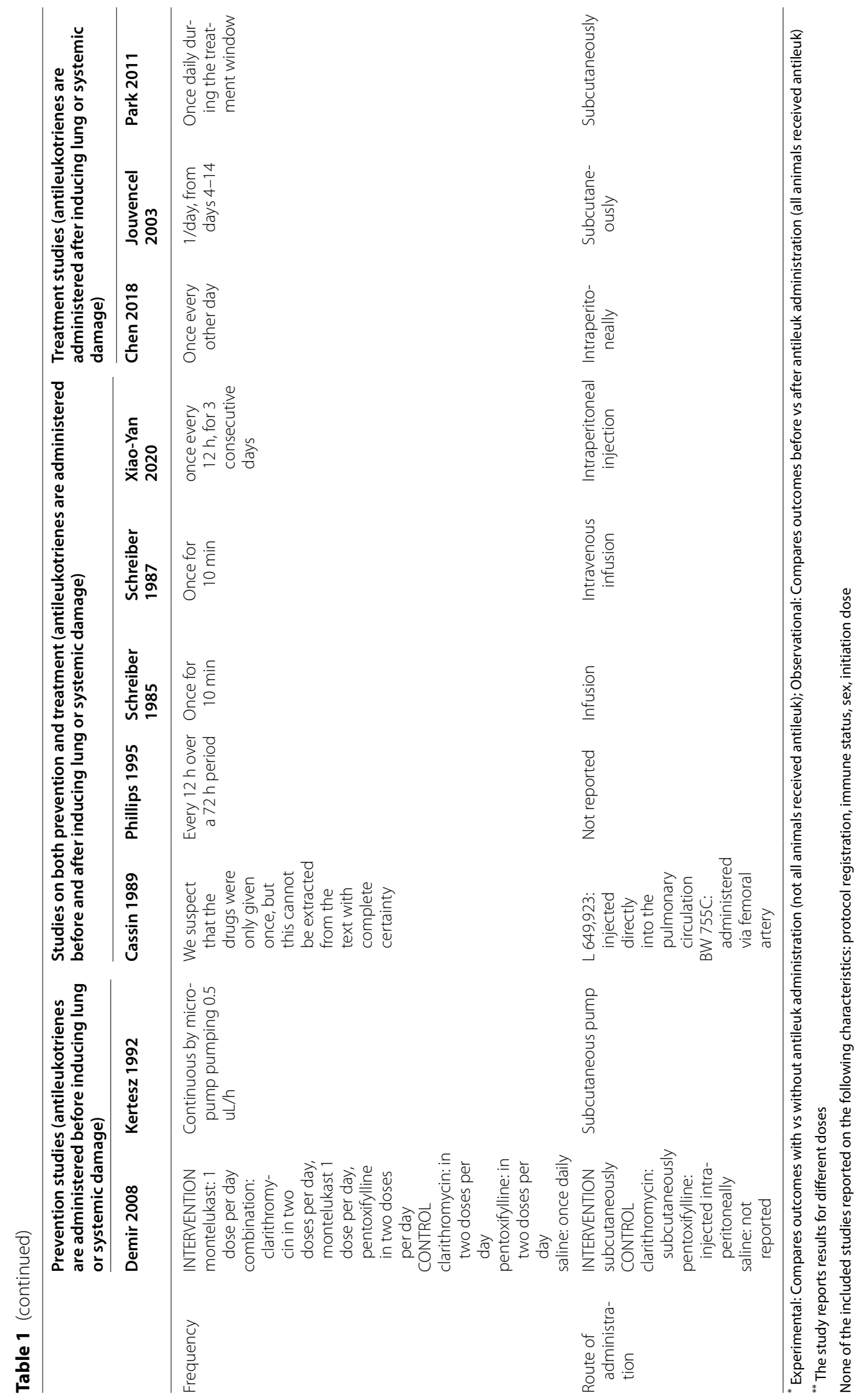




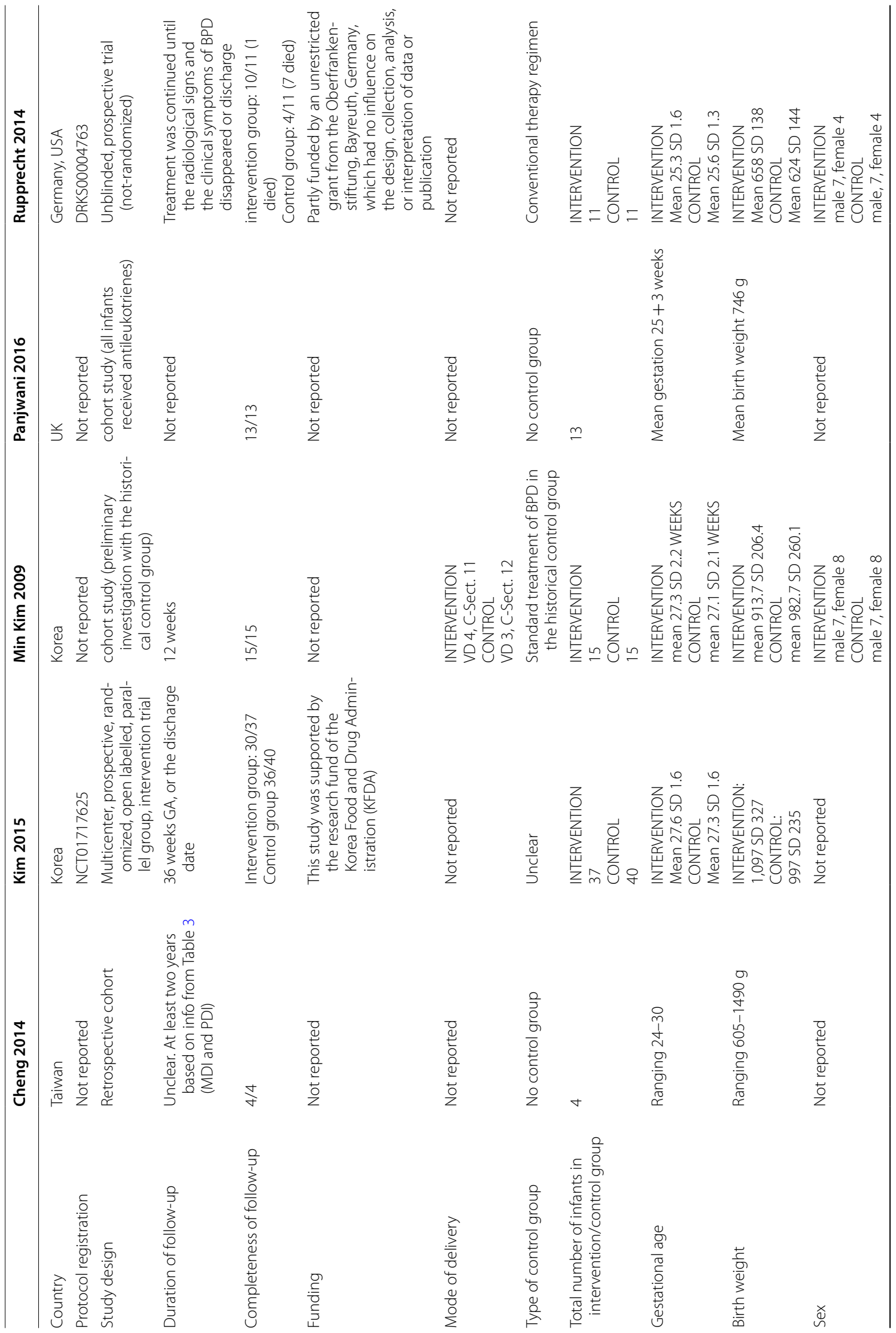




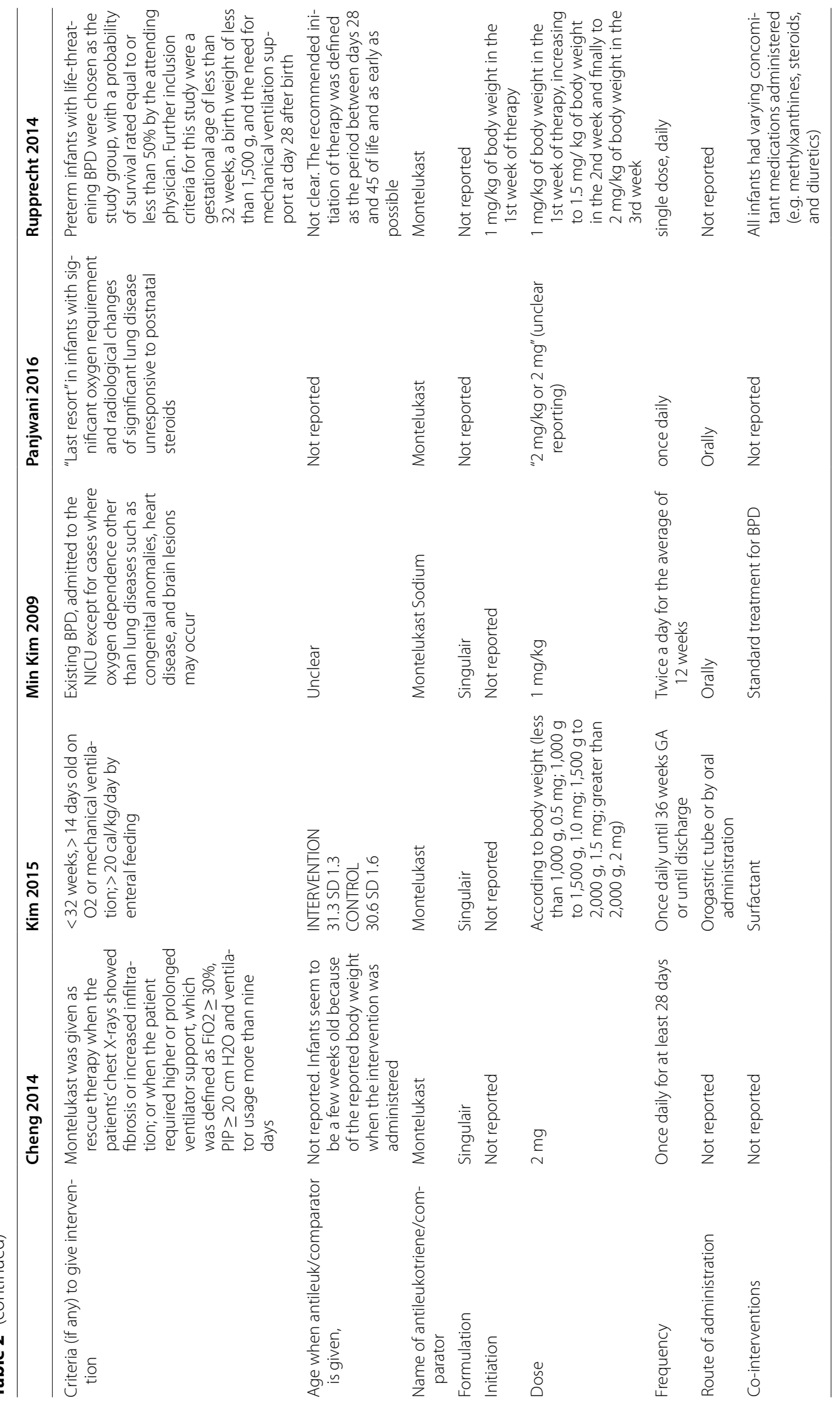




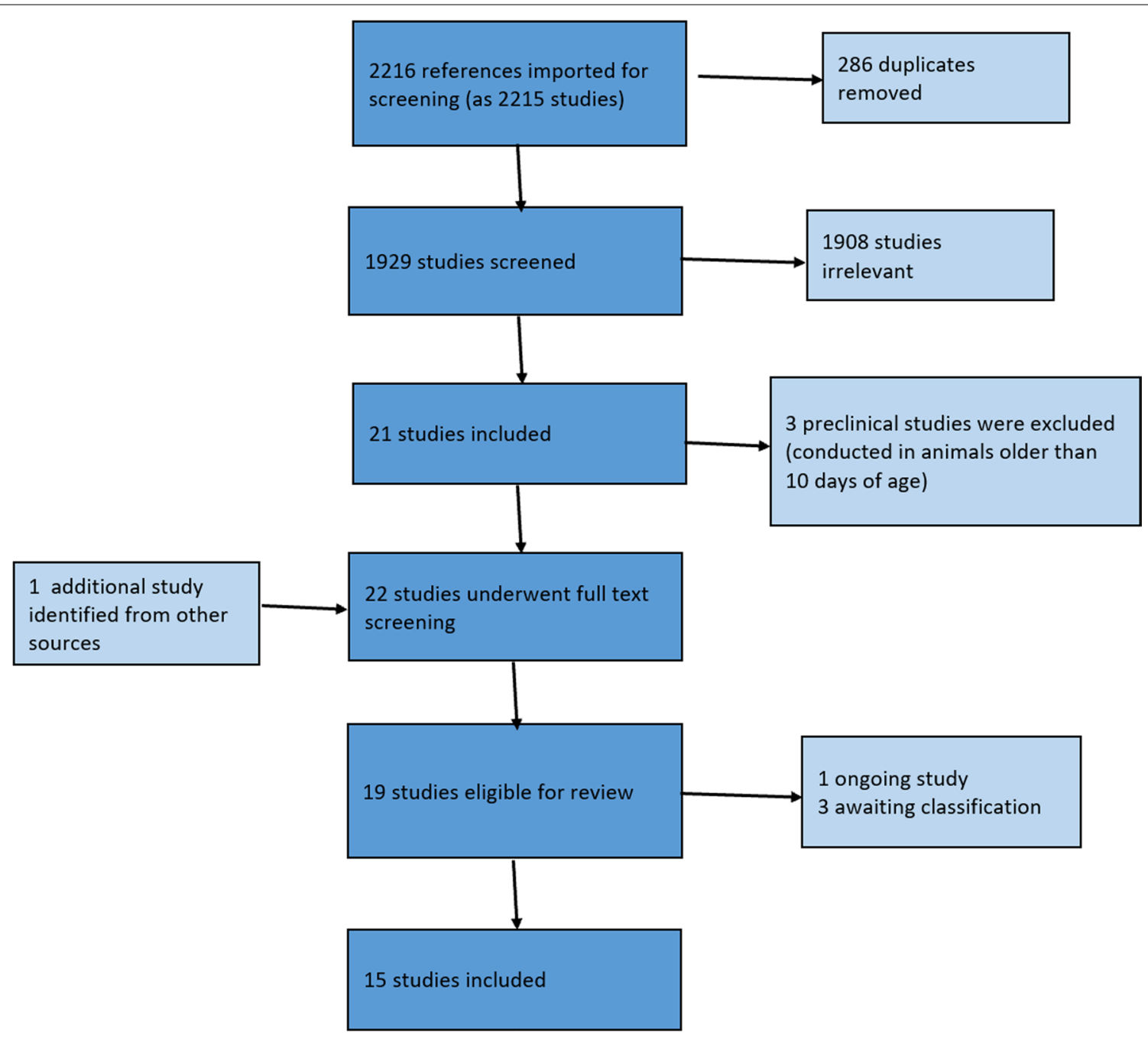

Fig. 1 PRISMA flow diagram

used to house the animals randomly within the animal room were not reported. Blinding of the investigators was also not reported. Only one study [37] was assessed as at low risk of detection bias as they reported that "all morphometric assessments were made blindly by the same observer (except for the bronchial alveolar attachments)".

All studies had unclear or higher risk of reporting bias as their protocol was not available. One study reported that data for some outcomes were not shown [42] and thus had high risk of bias. The animal studies appeared free from other sources of biases.

\section{Clinical studies}

Details of our risk of bias assessments for the randomized and non-randomized studies are presented in Tables 4 and 5 , respectively.
None of the included clinical studies were assessed to have low risk of bias. The single included RCT had an overall risk of bias assessed as "some concerns" [46] due to missing information about the randomization process; unclear description of the infants that were not included in the final analysis and because it was unclear whether the outcome assessors were blinded. The cohort study did not clearly define inclusion and exclusion criteria and was therefore assessed to have serious risk of bias [48]. The study by Rupprecht et al. [47] was scored with an overall critical risk of bias because of confounding as the control group consisted of children whose parents provided informed consent for participation as a control group patient but not for administration of the medication montelukast. The reasons for only allowing the child into the control group 


\begin{tabular}{|c|c|c|c|c|c|c|}
\hline 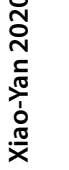 & 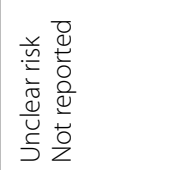 & 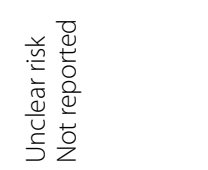 & 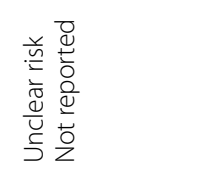 & 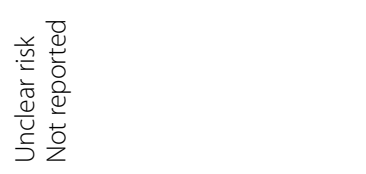 & 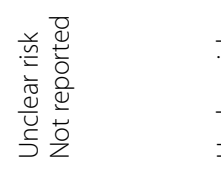 & 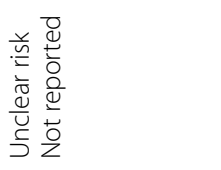 \\
\hline & 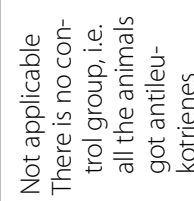 & 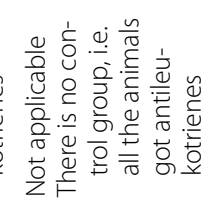 & 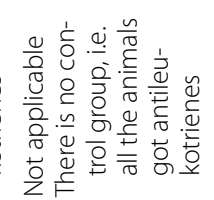 & 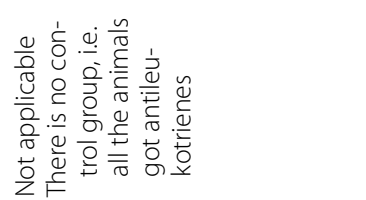 & 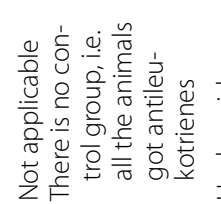 & 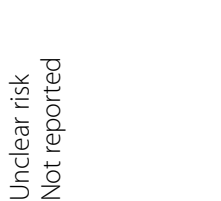 \\
\hline 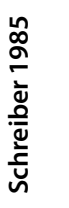 & 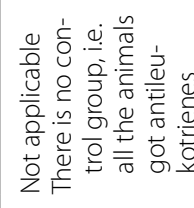 & 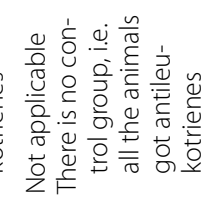 & 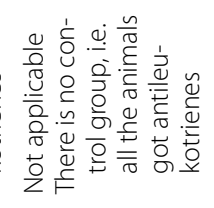 & 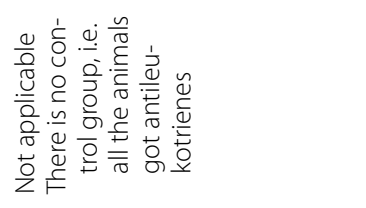 & 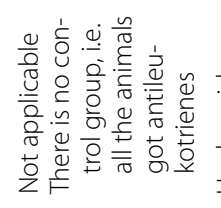 & 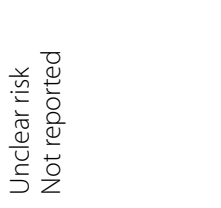 \\
\hline$\frac{\stackrel{n}{\alpha}}{\frac{n}{n}}$ & 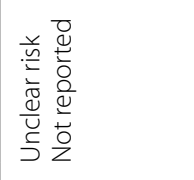 & 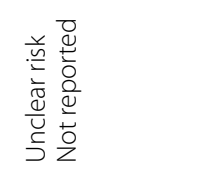 & 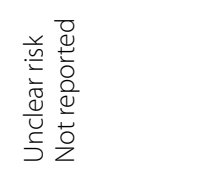 & 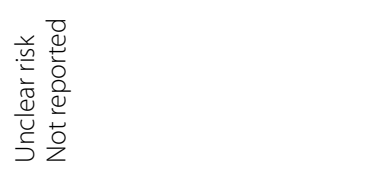 & 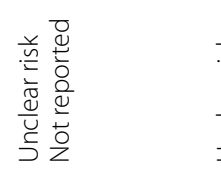 & 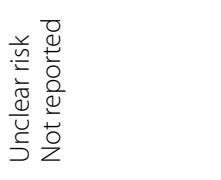 \\
\hline & 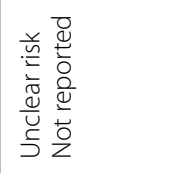 & 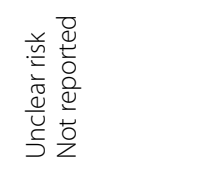 & 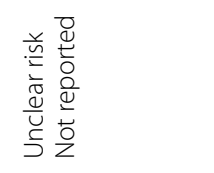 & 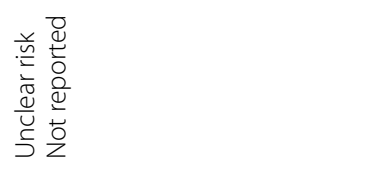 & 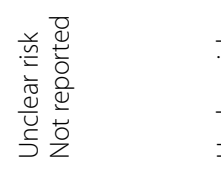 & 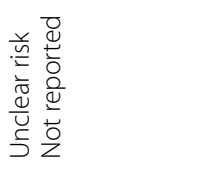 \\
\hline 命 & 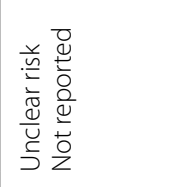 & 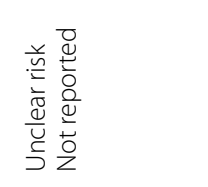 & 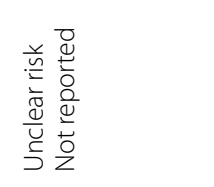 & 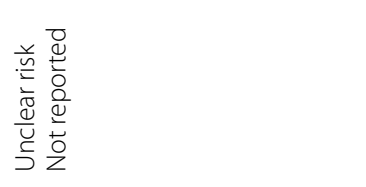 & 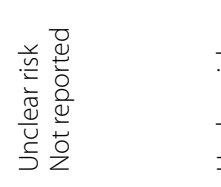 & 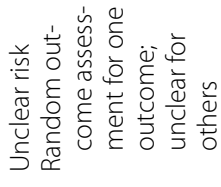 \\
\hline 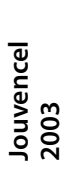 & 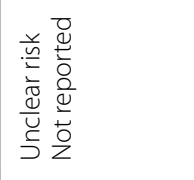 & 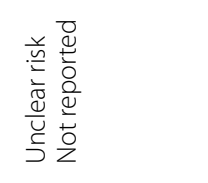 & 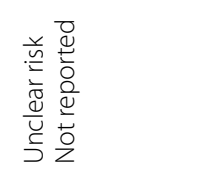 & 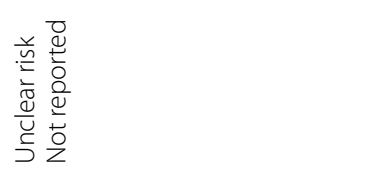 & 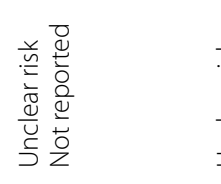 & 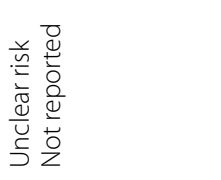 \\
\hline هั: & 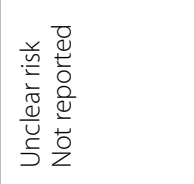 & 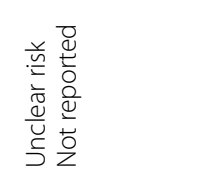 & 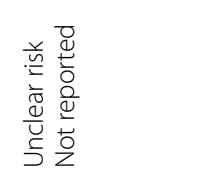 & 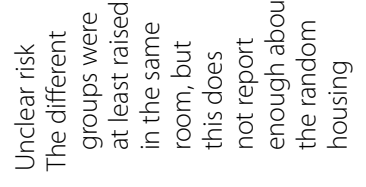 & 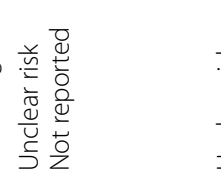 & 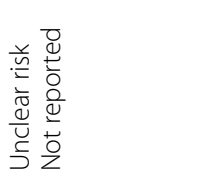 \\
\hline & 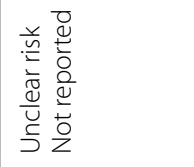 & 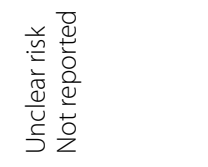 & 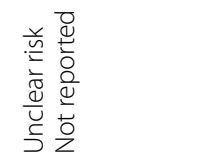 & 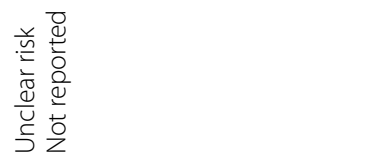 & 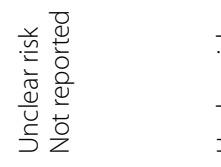 & 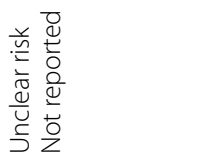 \\
\hline 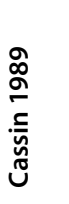 & 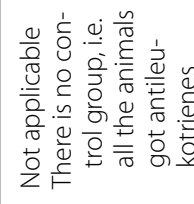 & 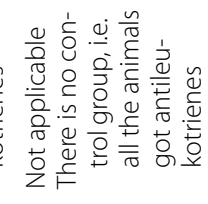 & 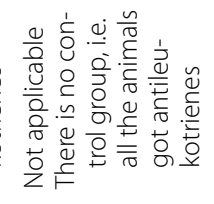 & 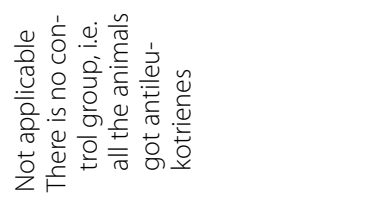 & 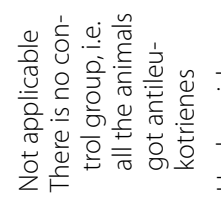 & 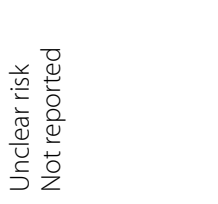 \\
\hline & 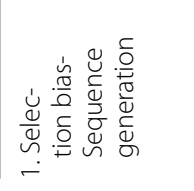 & 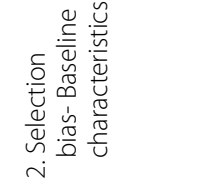 & 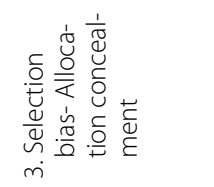 & 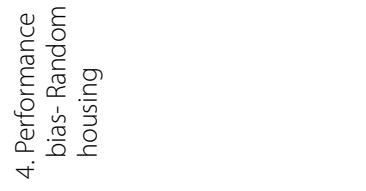 & 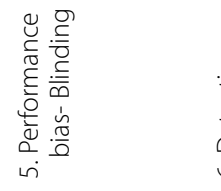 & 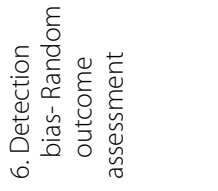 \\
\hline
\end{tabular}




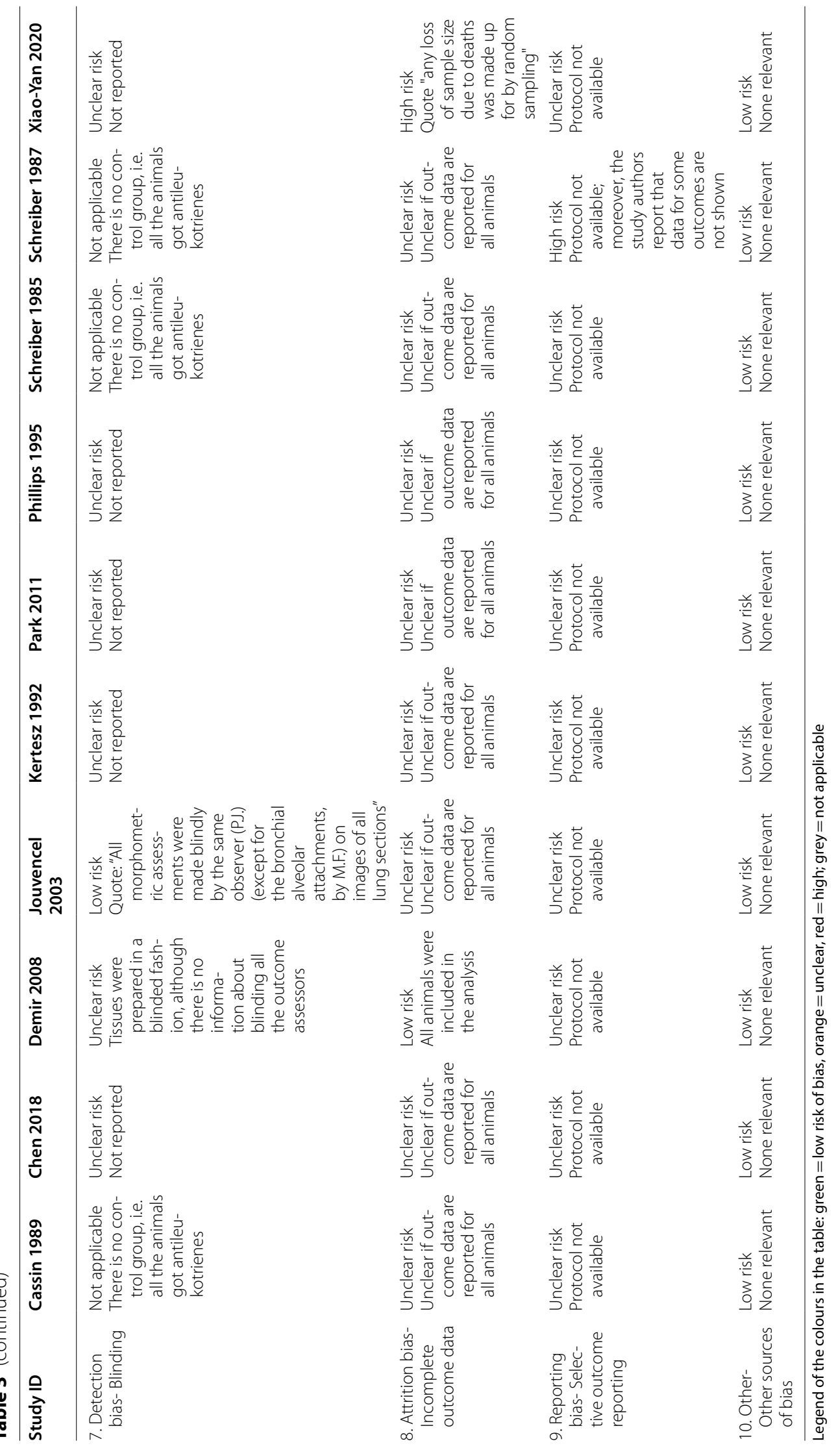


Table 4 Risk of Bias assessment with Rob 2.0 tool for the included RCT

\begin{tabular}{|c|c|c|c|c|c|c|}
\hline & $\begin{array}{l}\text { Randomization } \\
\text { process }\end{array}$ & $\begin{array}{l}\text { Deviations } \\
\text { from intended } \\
\text { interventions }\end{array}$ & $\begin{array}{l}\text { Missing outcome } \\
\text { data }\end{array}$ & $\begin{array}{l}\text { Measurement of the } \\
\text { outcome }\end{array}$ & $\begin{array}{l}\text { Selection of the } \\
\text { reported results }\end{array}$ & Overall risk of bias \\
\hline Kim 2015 & some concerns $^{\mathrm{a}}$ & some concerns ${ }^{b}$ & low & some concerns $^{d}$ & low & some concerns \\
\hline \multicolumn{7}{|c|}{ a No information about allocation concealment, randomization of groups was performed using shuffled blocks of random numbers in Microsoft Office, Excel 2007} \\
\hline \multicolumn{7}{|c|}{${ }^{b}$ Unclear description of the infants that were not included in the final analysis } \\
\hline \multicolumn{7}{|c|}{${ }^{\mathrm{C}}$ Data appears to be complete. Attrition and exclusions were explained (not completely clear though) and accounted for } \\
\hline \multicolumn{7}{|c|}{${ }^{d}$ Unclear if outcome assessors were blinded } \\
\hline \multicolumn{7}{|c|}{ e Seems in accordance with protocol } \\
\hline
\end{tabular}

Table 5 Risk of Bias assessment with ROBINS-I tool for the included non-randomized studies

\begin{tabular}{|c|c|c|c|c|c|c|c|c|}
\hline & Confounding & $\begin{array}{l}\text { Selection of } \\
\text { participants } \\
\text { into the study }\end{array}$ & $\begin{array}{l}\text { Classification } \\
\text { of } \\
\text { interventions }\end{array}$ & $\begin{array}{l}\text { Deviations } \\
\text { from } \\
\text { intended } \\
\text { interventions }\end{array}$ & Missing data & $\begin{array}{l}\text { Measurement } \\
\text { of outcomes }\end{array}$ & $\begin{array}{l}\text { Selection of } \\
\text { the reported } \\
\text { results }\end{array}$ & $\begin{array}{l}\text { Overall risk of } \\
\text { bias }\end{array}$ \\
\hline $\begin{array}{l}\text { Rupprecht } \\
2014\end{array}$ & Critical $^{a}$ & Low & Low & Low & Low & Moderate $^{f}$ & Low & Critical \\
\hline Min Kim 2009 & Low $^{b}$ & Serious $^{c}$ & Low & Moderate $^{d}$ & Moderate & Moderate $^{g}$ & Moderate $^{h}$ & Serious \\
\hline Panjwani 2016 & $\begin{array}{l}\text { No informa- } \\
\text { tion }\end{array}$ & Serious $s^{i}$ & $\begin{array}{l}\text { No informa- } \\
\text { tion }\end{array}$ & $\begin{array}{l}\text { No informa- } \\
\text { tion }\end{array}$ & $\begin{array}{l}\text { No informa- } \\
\text { tion }\end{array}$ & No information & $\begin{array}{l}\text { No informa- } \\
\text { tion }^{j}\end{array}$ & Serious \\
\hline Cheng 2014 & $\begin{array}{l}\text { No } \\
\text { informationk }^{k}\end{array}$ & Critical $^{\prime}$ & Moderate $^{\mathrm{m}}$ & Low & $\begin{array}{l}\text { No informa- } \\
\text { tion }\end{array}$ & Moderate $^{n}$ & Moderate $^{\circ}$ & Critical \\
\hline
\end{tabular}

${ }^{a}$ The control group consisted of children whose parents provided informed consent for participation in this study (as a control group patient) but not for administration of the medication montelukast (controls 1-5, 8,and 9; Table 1); and children in whom the planned therapy scheme was not possible due to existing or arising contraindications for the study drug (4 children, phenobarbital therapy in controls $6,7,10$, and 11)

${ }^{b}$ No significant difference between groups regarding patients' characteristics

'There is no clear definition of inclusion and exclusion criteria

${ }^{d}$ The study does not specify the exact time for which montelukast was given and for how long co-interventions of the conservative treatment were given, which may lead to relevant differences in co-interventions

e Data appears to be complete, although no protocol was published and the study was not registered as a clinical study

f Outcome "Duration for mechanical ventilation" might be biased by unblinded outcome assessor

${ }^{g}$ Outcome "Need for mechanical ventilation" might be biased by unblinded outcome assessor

${ }^{h}$ There is a discrepancy between text of the results section and table about vomiting or diarrhea as an adverse effect

'The study uses historical cohort as comparator, there is no clear definition of inclusion criteria, exclusion criteria are not well-specified

j Only abstract is available

k Information about possible confounding is insufficient

' Historical cohort, no clear definition of inclusion and exclusion criteria, no control group

${ }^{m}$ Subjective inclusion criteria

n Outcomes 'hospital stay' and 'respiratory support (duration, days)' are subjective

${ }^{\circ}$ No protocol published

are not reported. This leads to critical risk of bias in the domain 'bias due to confounding. The infants in the control group could have been potentially sicker than those in the montelukast group, in which case the parents might not be willing to try a drug with unknown effects on their fragile child. Therefore, the reduced rate of mortality in the infants treated with montelukast could be markedly different from the true effect. The study by Panjwani et al. [49] had a serious risk of bias. The study used a historical cohort as comparator and there was no clear definition of their inclusion and exclusion criteria. The study by Cheng et al. [50] had an 
Table 6 Outcomes antileukotrienes animals

\begin{tabular}{|c|c|c|c|c|c|c|c|c|}
\hline & Chen 2018 & Demir 2008 & Jouvencel 2003 & Kertesz 1992 & Park 2011 & Phillips 1995 & Schreiber 1985 & Xiao-Yan 2020 \\
\hline Mortality & Not reported & See note 1 & See note 2 & See note 3 & See note 4 & See note 5 & Not reported & See note 6 \\
\hline Somatic growth & Not reported & See note 7 & See note 8 & Not reported & Not reported & Not reported & Not reported & Not reported \\
\hline $\begin{array}{l}\text { Lung volume to } \\
\text { body weight }\end{array}$ & See note 9 & Not reported & See note 10 & See note 11 & Not reported & Not reported & Not reported & Not reported \\
\hline Lung histology & See note 12 & See note 13 & See note 14 & Not reported & See note 15 & See note 16 & Not reported & Not reported \\
\hline $\begin{array}{l}\text { Inflammation mark- } \\
\text { ers for lungs }\end{array}$ & See note 17 & Not reported & Not reported & See note 18 & Not reported & See note 19 & Not reported & Not reported \\
\hline Lung injury & See note 20 & Not reported & Not reported & Not reported & Not reported & Not reported & Not reported & Not reported \\
\hline $\begin{array}{l}\text { Airway hyper- } \\
\text { responsiveness, } \\
\text { fibrosis and } \\
\text { smooth muscle } \\
\text { actin expression }\end{array}$ & Not reported & See note 21 & Not reported & Not reported & Not reported & Not reported & Not reported & Not reported \\
\hline Behavioral tests & Not reported & Not reported & Not reported & Not reported & Not reported & Not reported & Not reported & See note 22 \\
\hline $\begin{array}{l}\text { Pulmonary vascular } \\
\text { resistance }\end{array}$ & Not reported & Not reported & Not reported & Not reported & Not reported & Not reported & See note 23 & Not reported \\
\hline
\end{tabular}

None of the included studies reported on the following outcomes: Harms, lung function, markers for apoptosis, Fulton index, arterial wall structure

Schreiber 1987 and Cassin 1989 are not listed in the table as they reported none of the outcomes specified in our review

Intervention: montelukast 0/10 combination: 0/6 Control: clarithromycin 0/8 pentoxifylline 0/8 placebo 0/6

Intervention 0/12. Control 0/12

Experiment 1: not reported Experiment 2: intervention: the percent mortality of the rabbits at any given number of hours of exposure to $>95 \%$ (\%) (48 h: $0 ; 60 \mathrm{~h}: 43$; $84 \mathrm{~h}: 65108 \mathrm{~h}: 88132 \mathrm{~h}: 88)$. Control: experiment 2: The percent mortality of the rabbits at any given number of hours of exposure to >95\% (\%) (48 h: $0 ; 60 \mathrm{h:} 41 ; 84 \mathrm{h:}$ $59108 \mathrm{~h}: 79132 \mathrm{h:} \mathrm{100)}$. There were no significant differences at any time between the ICl and the control group

There was no mortality among study animals

In the prevention study (normoxia), 3 out of 19 and 1 out of 6 pups died in the antileukotriene and control group, respectively. In the treatment study (hyperoxia), 3 out of 22 and 0 out of 6 pups died in the antileukotriene and control group, respectively

This outcome cannot be calculated because "any loss of sample size due to deaths was made up for by random sampling

Intervention: montelukast: Me 13 SD 0.6 g; combination: Me 10.1 SD 1.1 g;:control clarithromycin: Me 9.3 SD 0.7 g; pentoxifylline: Me 9.2 SD 3.2; placebo: Me 11.6 SD

$2.2 \mathrm{~g}$; montelukast vs placebo $\mathrm{p}=0.07$; montelukast vs. clarithromycin $p<0.0001$; montelukast vs. pentoxifylline $p=0.0019 ;$ combination vs. placebo $p=0.1661$

Intervention: mean 28.8 SD 0.5(g) (not relevant); control: Mean 28.5 SD $0.4 \mathrm{~g}$ (not relevant)

LW/BW intervention: Not reported, $\rightarrow$ lung weight/body weight (LW/BW): It is impossible to extract the data due to wrong values on $y$-axis)

Intervention: Mean 5.3 SD $0.13(\mathrm{ml} / 100 \mathrm{~g})$ (not relevant) control: Mean 5.15 SD 0.13 (not relevant)

LW/BW intervention: not reported(Lung water expressed as lung wet weight to body weight ratios $0.1 \mu \mathrm{M} / \mathrm{kg} / \mathrm{h} \mathrm{ICl} 48$ h: 1.3 SD ?; $72 \mathrm{~h}: 5,7$ SD 0.2; 84 h: 7.6 SD 0,4 96 h: 7.5 SD $0.5 ; 1.0 \mu \mathrm{M} / \mathrm{kg} / \mathrm{h} \mathrm{ICl} 84 \mathrm{~h}: 7.5$ SD ?; $96 \mathrm{~h}: 6,6 \mathrm{SD} 0.3$ ). Control: lung wet weight: body weight ratios began to increase at $72 \mathrm{~h}$ and continued to increase slowly after 84 and $96 \mathrm{~h}$ of hyperoxic exposure. No differences between intervention and control group (Fig. 3b) Control Lung water expressed as lung wet weight to body weight ratios control 48 h: 1.7 SD ?; 72 h: 5.4 SD 0.2; 84 h: 6.2 SD 0.496 h: 6.3 SD 0.5

Intervention: mean linear intercept (MLI): 93 SD .5; radial alveolar count (RAC) mean: 4.28 SD 0.24 -both p < 0.01 vs hyperoxia model. Control: mean linear intercept (MLI): 130 SD 7.7; radial alveolar count (RAC): 1.94 SD 0.1

Intervention: alveolar surface area (\%): group 3 montelukast Me 41.6 SD 4.8; group 5 combination: Me 64.0 SD 3; control: alveolar surface area (\%); clarithromycin Me 50.9 SD 4.2; pentoxifylline Me 59.4 SD 6.8; placebo Me 50.2 SD 10.4. montelukast vs. placebo $p=0.0389$ montelukast vs. clarithromycin $p=0.0005$ montelukast vs. pentoxifylline $p<0.0001$ combination vs. placebo $p=0.0093$

Intervention: surface density of parenchymal tissue mean 24.2 SD 1.2 (\%) (not relevant); mean linear chord length mean 53.3 SD 1.3 ( $\mu$ m) (not relevant) septal attachments (/mm bronchi) mean 29.1 SD 1.0 (not relevant). Control: surface density of parenchymal tissue mean 22.8 SD 0.5 (not relevant); mean linear chord length mean 52.7 SD 1.3(not relevant) septal attachments (/mm bronchi) mean31.7 SD 0.9 (not relevant)

Number of airspaces intervention: treatment group: (dose 40 mg/kg, P10-14): mean 20 SD 2. Prevention group (dose 40 mg/kg, p1-4): mean 19 SD 1 control treatment group (dose $0 \mathrm{mg} / \mathrm{kg}, \mathrm{P} 10-14)$ : mean 11 SD ? prevention: (dose $0 \mathrm{mg} / \mathrm{kg}, \mathrm{p} 1-4)$ : mean 6 SD 2

95\% oxygen + treatment: airspace (\%) (37.0 SD 6.0) neutrophils (No $\mathrm{mm}^{-2}$ ) (198 SD 10.9 (Different from 95\% O, control, p<0.05)) lung sections from pre-term guinea pig pups. $21 \%$ oxygen + treatment: airspace (\%) (43.5 SD 3.5) neutrophils (No $\mathrm{mm}^{2}$ ) (108 SD 8.5) lung sections from pre-term guinea pig pup

Intervention: relative TNF-a mRNA level mean: 2.0 SD 0.15; relative IL-6 mRNA level mean: 1.7 SD 0.06; relative IL-1 $\beta$ mRNA level: 1.9 SD 0.12 ; [not sure about p value, in the text: "Montelukast treatment significantly reduced the levels of TNF-a, IL-6, and IL- $1 \mathrm{~b}$ in the lung tissues of the BPD mice. control: relative TNF-a mRNA level mean: 3.3 SD 0.1; relative IL-6 mRNA level mean: 3.5 SD 0.2; relative IL-1 $\beta$ mRNA level mean: 2.9 SD 0.1

Intervention: Dose $0.1 \mu \mathrm{M} / \mathrm{kg} / \mathrm{h}$ : Total protein recovered from BAL mean ( $\mu \mathrm{g} / \mathrm{ml})$ ( $48 \mathrm{~h}$ and $72 \mathrm{~h}: 90$ SD 20; 84 h: 250 SD $120 ; 96 \mathrm{~h}: 330$ SD 40 ); PMNS represented as a percentage of the total (48 h: 0; 72 h: 1,3 SE 7; 84 h: 10 SE 5; 96 h: 18 SE 5) white cells recovered from BAL mean (\%); PMNs, represented as the absolute number recovered from BAL of the left lung ( $\times 100,000)(48 \mathrm{~h}$ and $72 \mathrm{~h}: 0.5 \mathrm{SE} \mathrm{0.2;} 84 \mathrm{~h}: 2,4 \mathrm{SE} 0.396 \mathrm{~h}: 2.9 \mathrm{SE} 0.3)$; 6-Keto-PGF 1 alfa the stable metabolite of PGl, in pg/ml (48 h: 71 SE no info; 72 h: 54 SE 28; 84 h: 144 SE 50; 96 h: 347 SE 463); TXB, the stable metabolite of TXA, in pg/ml mean (48 h: 115 SE no info; 72 h: 81 SE $19 ; 84$ h: 241 SE 121 ; $96 \mathrm{~h}: 207 \mathrm{SE} 22)$. Dose $1.0 \mathrm{uM} / \mathrm{kg} / \mathrm{h}$ : total protein recovered from BAL mean ( $\mu \mathrm{g} / \mathrm{ml})(84 \mathrm{~h}: 475 \mathrm{SD} 112 ; 96 \mathrm{~h}: 416 \mathrm{SD} 56)$; PMNS represented as a percentage of the total (48 h: 0; 72 h: no info; 84 h: 20 SE 4; 96 h: 14 SE 5) white cells recovered from BAL mean (\%); PMNs, represented as the absolute number recovered from BAL of the left lung ( $\times 100,000$ ) ( $48 \mathrm{~h}$ and $72 \mathrm{~h}$ : no info; 84 h: 2,9 SE 0.396 h: 2.1 SE 0.); 6-Keto-PGF, the stable metabolite of PGl, in pg/ml (48 h: no info; 72 h: no info; 84 h: 348 SE 32 ; 
Table 6 (continued)

$96 \mathrm{~h}: 315$ SE 32); TXB, the stable metabolite of TXA, in pg/ml mean (48 h: no info; $72 \mathrm{~h}:$ no info; 84 h: 211 SE 19; 96 h: 259 SE 37)

Control: total protein recovered from BAL mean $(\mu \mathrm{g} / \mathrm{ml})(48 \mathrm{~h}$ and $72 \mathrm{~h}: 90$ SD 20; $84 \mathrm{~h}: 392$ SD 61; $96 \mathrm{~h}: 420$ SD 56) PMNS represented as a percentage of the total ( $48 \mathrm{~h}: 0 ; 72 \mathrm{h:} \mathrm{1,3} \mathrm{SE} 8 ; 84 \mathrm{h:} 22$ SE 5; $96 \mathrm{~h}: 21$ SE 4) white cells recovered from BAL mean (\%); PMNs, represented as the absolute number recovered from BAL of the left lung ( $\times 100$ 000) ( $48 \mathrm{~h}$ and $72 \mathrm{~h}: 0.5 \mathrm{SE} 0.2 ; 84 \mathrm{~h}: 3,4 \mathrm{SE} 0,396 \mathrm{~h}: 3,5 \mathrm{SE}$ 0,2); 6-Keto-PGF, the stable metabolite of PGl, in (48 h: 71 SE no info; 72 h: 54 SE $28 ; 84$ h: $222 \mathrm{SE}$ 32; 96 h: 265 SE 44) TXB, the stable metabolite of TXA, in pg/ml mean (48 h: 115 SE no info; 72 h: 81 SE 19; 84 h: 241 SE 121; 96 h: 207 SE 22) pg/ml

95\% oxygen + treatment: neutrophil and eosinophil numbers and protein concentration in bronchoalveolar lavage fluid (BALF) neutrophils (10 4 ml-' BALF) 3.0: 1.85 SD 0.79 (Different from equivalent vehicle control, PcO.05.)) eosinophils (10 $6 \mathrm{ml}$-'BALF) 3,0:0.88 SD 0.37 protein (mg ml -'BALF) 3,0: 0.28 SD 0.127). 21\% oxygen + treatment: neutrophil and eosinophil numbers and protein concentration in bronchoalveolar lavage fluid (BALF) neutrophils(10 4 ml-'BALF) 3,0: 1.45 SD 1.56 eosinophils (10 $6 \mathrm{ml} \mathrm{-'BALF)} \mathrm{3.0:} 0.94$ SD 0.31(Different from equivalent vehicle control, PcO.05.) protein ( $\mathrm{mg} \mathrm{ml} \mathrm{-'BALF)3.0:} \mathrm{0.27} \mathrm{SD} \mathrm{0.08)}$

Intervention: oxidative stress malondialdehyde $1.4+-0.1 \mathrm{mcmol} / \mathrm{g}$ (mean, sd); SOD superoxide dismutase $22.0+-1 \mathrm{IU} / \mathrm{mg}$ (mean, sd). Control: oxidative stress malondialdehyde $1.9+-0.05 \mathrm{mcmol} / \mathrm{g}$ (mean, sd); SOD superoxide dismutase $16.5+1 \mathrm{IU} / \mathrm{mg}$ (mean, sd)

Degree of fibrosis absent /mild /moderate /marked Intervention: group 3 montelukast 0/1/6/3 group 5 combination: 4/2/0/0. Control: clarithromycin 0/1/3/4 pentoxifylline 2/2/4/0 placebo0/2/3/1. Actin score (density x intensity) Intervention: group 3 montelukast: 5 (2-9) group 5 combination: $0(0-1)$ Control: clarithromycin 7.5 (2-9) pentoxifylline 1.5 (0-6) placebo 7 (2-12)

Compared with the PVL group, the escape latency of the rats in the Pran group was shortened $(p<0.05)$ (Table 2$)$. On the 5 th day of the experiment, there was a statistically significant difference in the number of times the rats in each group crossed the platform $(F=12.59, p<0.001)$. Compared with the PVL group, the number of times $(1.86 \pm 0.23)$ of rats in the Pran group crossed the platform increased $(p<0.05)$

Intervention: me 44.0 SD 7.0 in mmHg 1-1 $\mathrm{min}^{-1} \mathrm{~kg}^{-1}$. Control: me 70.3 SD 15.5 ( $p<0.05$ vs hypoxia +FPL 57,231) in mmHg1-1 $\mathrm{min}^{-1} \mathrm{~kg}^{-1} \mathrm{p}=0.0086$

overall critical risk of bias as a historical cohort design was used without clear inclusion and exclusion criteria.

\section{Effects of the interventions}

Meta-analysis of the clinical and the animal studies was not deemed feasible for any of the outcomes since they were reported by too few studies with highly variable designs and were assessed with outcome measures which could not be meaningfully pooled.

\section{Animal studies}

Table 6 shows the list of the outcomes reported by each study.

Four controlled studies reported on mortality and found no significant effect [36, 37, 40, 44]; two controlled studies reported on growth [36, 37]; no significant effect was found in either study between combination treatment (montelukast, clarithromycin and pentoxifylline combination) versus placebo.

We made the post hoc decision to include the reported outcome 'lung weight to body weight ratio', in addition to our prespecified outcome lung volume to body weight. No statistically significant difference was found in the three studies reporting on either of the two outcomes [37, 39, 44].

Five experimental studies assessed lung histology, reporting on different outcomes, i.e. radial alveolar count [39], alveolar surface area [36], parenchymal tissue [37], number of airspaces [40] and percentage of airspace [43]. No firm conclusions could be drawn (see Table 6 for more information).
Three studies reported on inflammation markers for lungs [39, 43, 44]. Two studies [43, 44] reported on polymorphonuclear leukocytes and protein in bronchoalveolar fluid. Phillips et al. [43] showed a reduction in the number of neutrophils and protein in the treated hypoxia group and in eosinophils in the treated normoxia group. The study by Chen et al. [39] detected a reduction in the concentration of other inflammation markers in the lung tissue of BPD mice.

Lung injury was reported in one study in which montelukast treatment decreased malondialdehyde levels and enhanced superoxide dismutase activity in the lung tissues of the BPD mice [39].

The study by Demir et al. [36] was the only study to report fibrosis and smooth muscle actin expression. They did not detect an effect of montelukast alone versus placebo; the combination treatment (montelukast, clarithromycin and pentoxifylline combination) did result in a lower actin score compared to the placebo group.

Only one study reported on behavioral tests, the Morris water maze experiment [38]. There was an improvement in escape latency in the pranlukast group and the number of times rats in the pranlukast group crossed the platform in the maze increased.

The study by Schreiber et al. [41] found a decrease in pulmonary vascular resistance in lambs after antileukotriene infusion.

None of the animal studies reported on harms, lung function, markers for apoptosis, Fulton index or arterial wall structure. 


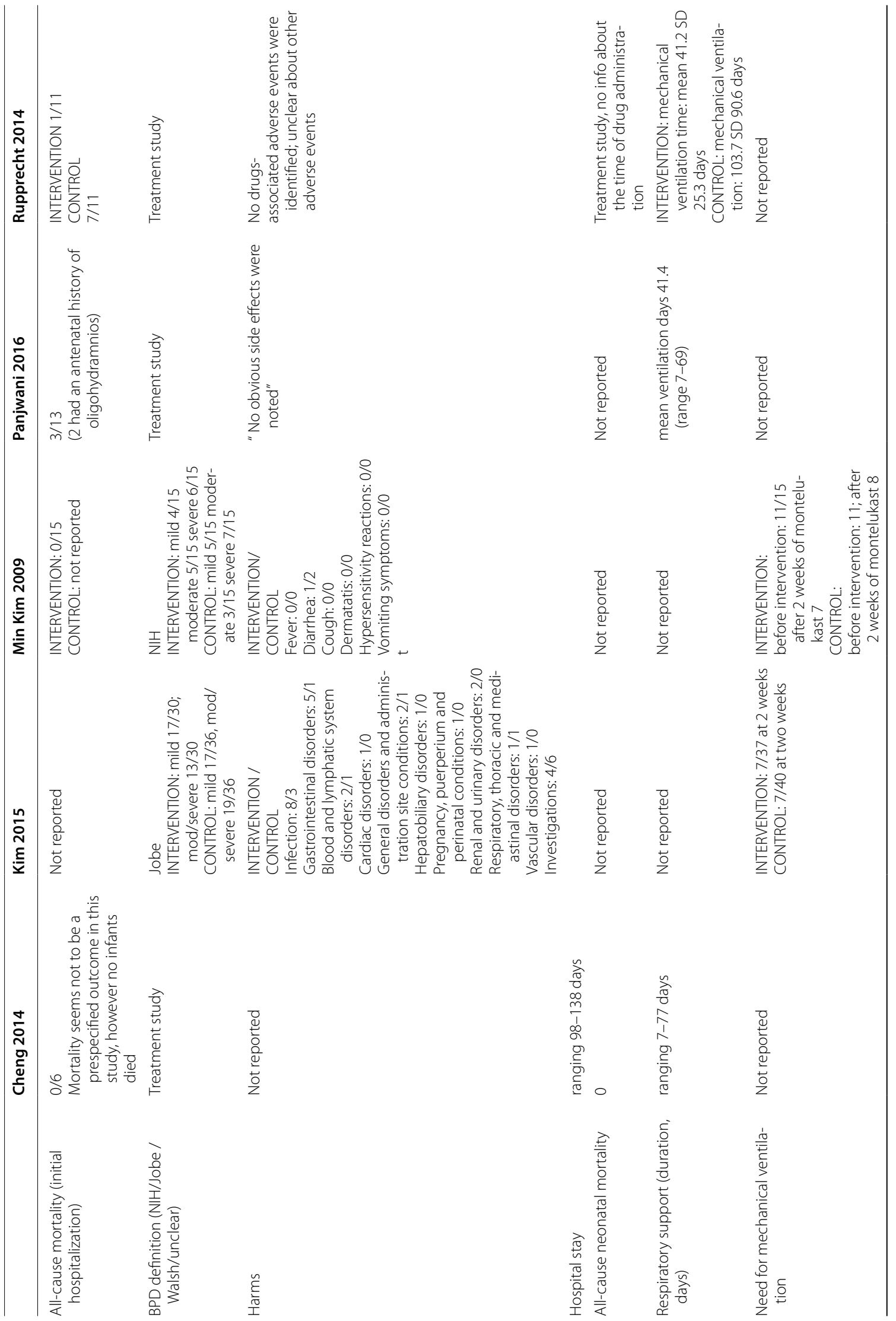




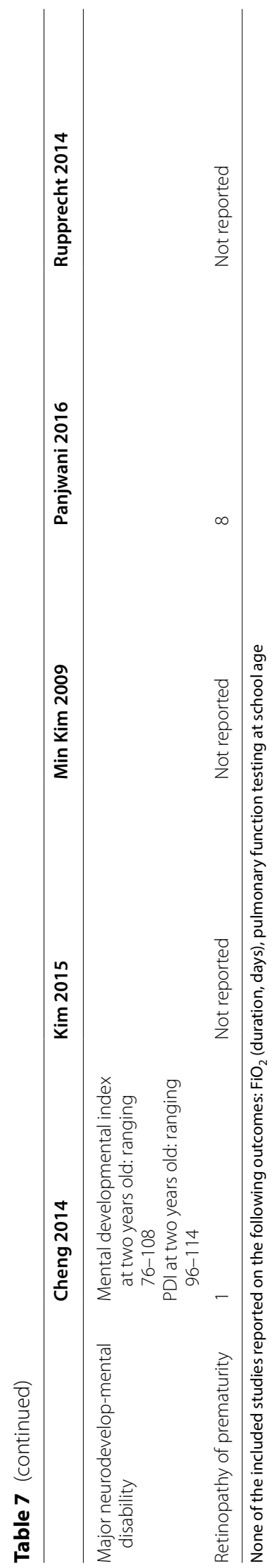




\section{Clinical studies}

Outcomes for the randomized trial [46] and the four non-randomized [47-50] clinical studies are reported in Table 7.

Two clinical studies reported on all-cause mortality [47, 48]; only one study [47], non-randomized, reported on all-cause mortality for both the intervention and the control group and found a significant reduction in allcause mortality in the montelukast group.

The two studies that reported on frequency and the severity classification of BPD showed no relevant difference between case and control group [46, 48].

The occurrence of adverse events did not differ between intervention and control groups in either the randomized trial [46] or the observational study by Kim [48]. It was unclear whether the other three studies had planned to report adverse events, but they did not.

Rupprecht et al. [47] did not provide information about the timing of drug administration and therefore all-cause neonatal mortality could not be extracted from the study for our pre-defined time point. Kim et al. [46], Panjwani et al. [49] and Min Kim et al. [48] did not report all-cause neonatal mortality.

Rupprecht et al. [47] reported a significantly shorter duration of respiratory support in the group receiving montelukast compared to controls (41.2 \pm 25.3 vs. $103.7 \pm 90.6$ days $)$.

Two studies reported on mechanical ventilation and found no differences $[46,48]$.

None of the included studies reported on fraction of inspired oxygen duration or pulmonary function testing at school age.

\section{GRADE assessment}

The certainty of the evidence was "very low" for all outcomes because of imprecision and high risk of bias in multiple other domains, both in clinical and animal studies.

\section{Discussion}

\section{Summary of main findings}

In this systematic review, we aimed to systematically assess the effects of antileukotrienes for the prevention and treatment of chronic lung disease in very preterm newborns. We included five clinical studies and ten animal studies. The clinical studies consisted of one RCT and four non-randomized studies. These five clinical studies and three of the animal studies examined the leukotriene antagonist montelukast. We did not find it meaningful to pool results because of the differences in study design and the high overall risk of bias. Drawing definitive conclusions on basis of the existing evidence is thus not possible.

\section{Overall completeness and applicability of evidence}

The animal studies had an overall unclear risk of bias due to poor reporting. None of the experimental studies reported on sequence generation, baseline characteristics, allocation concealment, random housing, blinding of the caregivers or random outcome assessment. Only Jouvencel [37] reported adequately on blinding of the outcome assessor, and Demir [36] on completeness of the outcome data, whereas the other studies lacked information. The fact that a protocol was not available for any of the animal studies is also noteworthy. This leads to unclear risk of reporting bias and poor transparency in general. In the case of Schreiber [42] it was also mentioned that data for some outcome was not shown, which causes a high risk of bias for outcome reporting. We classified Phillips [43] as assessing both prevention and treatment effects, as we considered the pups treated with antileukotriene in normoxic conditions as the prevention group and the pups with hyperoxia as the treatment group. Seven studies had an experimental design, i.e. the animals were exposed to two or more different interventions, whereas in the remaining three studies all animals received the same intervention and were therefore defined as observational.

Only one study reported outcome data following hospital discharge [50]. Kim et al. [46] is the first prospective study of montelukast for very preterm infants. Min Kim [48] was a cohort study with a historical control group. The study did not provide a clear definition of the inclusion and exclusion criteria, which leads to a serious risk of bias in the selection of participants.

\section{Relation to other research}

A study from 2019 evaluated incidence trends of neonates born very preterm in 11 high-income countries and reported increased BPD rates in most countries [51]. Main reasons for this trend include the increased survival of extremely preterm infants and active resuscitation at lower gestational age. Additional interventions are needed to prevent and treat this condition. Of note, montelukast is already being used as a drug in infants with BPD [52]. Interestingly, in this leaflet released/ published by American Thoracic Society, montelukast is only listed as an anti-inflammatory medicine for children with BPD.

The administration of off-label drugs in neonates are a universal problem. This forces the neonatologist to rely mostly on clinical experience, expert consensus and data extrapolation from patients other than neonates when deciding upon drug choice and dosage [53]. This supports the need for additional high 
quality research on this topic. We identified one ongoing observational study that aims to explore the effects of montelukast on very low birth weight infants with BPD [35]. The planned sample size, 200 infants, is considerably larger than the clinical studies performed so far and might allow to better explore potential harms of antileukotrienes administration. However, a randomized design would be preferable to assess the efficacy.

\section{Strengths and weaknesses of our review}

This is the first systematic review that explores the evidence base of antileukotrienes in very preterm infants in both clinical and animal studies. The review has several strengths. We conducted a comprehensive search with no date or language restrictions. We had studies translated from Mandarin [38] and Korean to English [48]. Further, all the potentially eligible titles and abstracts were screened independently by two authors, as were data extraction and the assessment of risk of bias. We used the most recent and validated tools to assess risk of bias in trials, non-randomized studies and animal studies.

Limitations include our arbitrary definition of neonate animals, i.e. up to 10 days of life. As the definition of a newborn infant (up to 28 days of life) is not based on a specific developmental phase or level of maturation it is not possible to identify a corresponding age in animal models. Further, we did not find any metaanalyses feasible. To retrieve additional information we contacted the authors of two conference abstracts and of the registered protocol we identified in our searches, however, we did not receive any response. Therefore, we could not include these studies and this restriction, though outside of our control, is a potential source of bias.

\section{Implications for research and practice}

Refining the existing models to recapitulate the pathology at play in the infants is an urgent matter in order to better evaluate new interventions for BPD [54]. Most animal experiments are carried out to gather information about health in humans and aim to investigate new interventions that are intended for future use in humans. Differences in outcomes in animals and humans are partly due to fundamental biological differences. However, other factors such as for instance design, conduct and reporting play an equally important role [55]. Future animal studies should be designed with higher quality and aim to minimize potential sources of bias, as described in the SYRCLE tool [28]. The registration of the protocols of animal studies in free databases such as https://preclinicaltrials.eu/ should become a standard practice and become a formal requirement placed by journals to publish such studies, as is already commonly done for the clinical studies. Similarly, an appropriate randomization should be performed to ensure that animals in each group are in the same housing conditions (e.g. temperature, humidity, light, noise, odors) and to avoid that researchers subjectively select which animals and samples to be used for outcome assessment. Finally, animal studies should clearly report how many animals were used in each step of the experiment, from inclusion to reporting of all outcomes, so that attrition bias can be assessed. As all the animal studies included in this review failed to address these key components in conducting and reporting, the translational value is extremely limited.

Seven of the ten animal studies used an antileukotriene other than montelukast. We speculate that, unless justified by species or pharmacokinetics characteristics, in future animal studies only montelukast should be administered, as only this drug has been used in all clinical studies so far, including the large ongoing study. When choosing outcomes, the focus should be on those with clinical relevance, such as mortality, improved respiratory function and harms.

Future clinical studies should preferably be designed as large, high quality RCTs [56]. New trials are necessary as the harms are not negligible. The findings of the ongoing study [35] with a planned sample size of 200 infants are not available yet. Multicenter RCTs would be an option in order to reach a sufficient sample size. Just as for animal studies, the focus should be on clinically relevant outcomes.

\section{Conclusions}

Based on the available evidence, no reliable conclusions about the clinical relevance of antileukotriene administration to very preterm infants can be drawn. Large randomized trials that focus on outcomes relevant to patients and their families, including long-term outcomes, are needed. Animal studies should prioritize montelukast over other antileukotrienes and minimize risks of bias. 


\section{Appendix}

Matthias Bank, Librarian / Information specialist

Library \& ICT, Faculty of Medicine

Lund University

Matthias.Bank@med.lu.se

LUND UNIVERSITY Faculty of Medicine

\section{SEARCH REPORT}

\section{PROJECT INFORMATION}

REQUEST DATE

NAME

INSTITUTION/ORGANISATION

20200908

Matteo Bruschettini

Cochrane Sweden

PROJECT TIME FRAME - DESIRED DATE OF DELIVERY

\section{September 15}

\section{DESCRIBE RESEARCH QUESTION AND PURPOSE WITH THE SEARCH}

Leukotriene receptor antagonists for the prevention and treatment of chronic lung disease in preterm infants. Systematic Review

\section{SEARCH TERMS}

PROVIDE SEARCH TERMS ACCORDING TO PICO(S), PEO OR IN FREE ORDER

\begin{tabular}{ll}
\hline Patient / population / problem & Preterm infants \\
\hline Intervention & Leukotriene \\
\hline Comparison / control & \\
\hline Outcome & \\
\hline Studytype & No studytype limitation \\
\hline
\end{tabular}


REFERENCES TO KEY ARTICLES - EXAMPLES OF YOU WANT TO HAVE INCLUDED IN THE SEARCH RESULT (3-5 ARTICLES)

DESIRED DELIVERY FORMAT (ENDNOTE, WORD, PDF)

EndNote

SEARCH STRATEGIES

Date of all searches September 15, 2020

PubMed

((infant, newborn[MeSH Terms]) OR (infant, premature[MeSH Terms])) OR ((newborn*[Title/Abstract] OR new born[Title/Abstract] OR new borns[Title/Abstract] OR newly born[Title/Abstract] OR baby*[Title/Abstract] OR babies[Title/Abstract] OR premature[Title/Abstract] OR prematurity[Title/Abstract] OR preterm[Title/Abstract] OR pre term[Title/Abstract] OR low birth weight[Title/Abstract] OR low birthweight[Title/Abstract] OR VLBW[Title/Abstract] OR LBW[Title/Abstract] OR infant[Title/Abstract] OR infants[Title/Abstract] OR infantile[Title/Abstract] OR infancy[Title/Abstract] OR neonat*[Title/Abstract])) AND ((C((montelukast) OR (zafirlukast)) OR (pranlukast)) OR ("leukotriene antagonists"[MeSH Terms])) OR ("leukotriene receptor antagonist" OR leukotriene OR leukotriene antagonist OR Singulair OR zileuton OR verlukast OR Pobilukast OR Cinalukast))

550 records

Embase(Elsevier)

'prematurity'/exp OR prematurity OR 'infant'/exp OR infant OR (newborn*:ab,ti OR 'new born':ab,ti OR 'new borns':ab,ti OR 'newly born':ab,ti OR baby*:ab,ti OR babies:ab,ti OR premature:ab,ti OR prematurity:ab,ti OR preterm:ab,ti OR 'pre term':ab,ti OR 'low birth weight':ab,ti OR 'low birthweight':ab,ti OR vlbw:ab,ti OR lbw:ab,ti OR infant:ab,ti OR infants:ab,ti OR infantile:ab,ti OR infancy:ab,ti OR neonat*:ab,ti)

AND

'montelukast'/exp OR montelukast OR 'leukotriene receptor blocking agent'/exp OR 'leukotriene'/exp OR leukotriene OR 'singulair'/exp OR singulair OR 'zafirlukast'/exp OR zafirlukast OR 'pranlukast'/exp OR pranlukast OR 'zileuton'/exp OR zileuton OR 'verlukast'/exp OR verlukast OR 'pobilukast'/exp OR pobilukast OR 'cinalukast'/exp OR cinalukast AND [embase]/lim NOT ([embase]/lim AND [medline]/lim) 411 records 


\section{CINAHLComplete(Ebsco)}

(infant or infants or infantile or infancy or newborn* or "new born" or "new borns" or "newly born" or neonat* or baby* or babies or premature or prematures or prematurity or preterm or preterms or "pre term" or premies or "low birth weight" or "low birthweight" or VLBW or LBW) AND (montelukast OR zafirlukast OR pranlukast OR "leukotriene antagonists" OR "leukotriene receptor antagonist" OR leukotriene OR leukotriene antagonist OR Singulair OR zileuton OR verlukast OR Pobilukast OR Cinalukast)

\section{4 records}

\section{Web of Science}

(infant or infants or infantile or infancy or newborn* or "new born" or "new borns" or "newly born" or neonat* or baby* or babies or premature or prematures or prematurity or preterm or preterms or "pre term" or premies or "low birth weight" or "low birthweight" or VLBW or LBW) AND

(montelukast OR zafirlukast OR pranlukast OR "leukotriene antagonists" OR "leukotriene receptor antagonist" OR leukotriene OR leukotriene antagonist OR Singulair OR zileuton OR verlukast OR Pobilukast OR Cinalukast)

1101 records

\section{Cochrane CENTRAL}

MeSH descriptor: [Infant, Newborn] explode all trees

OR

MeSH descriptor: [Infant, Premature] explode all trees

OR

MeSH descriptor: [Infant, Very Low Birth Weight] explode all trees

OR

infant OR infants ORinfantile OR infancy OR newborn* OR "new born" OR "new borns" or "newly born" or neonat* or baby* or babies or premature or prematures or prematurity or preterm or preterms or "pre term" or premies or "low birth weight" or "low birthweight" or VLBW or LBW or ELBW or NICU

AND

MeSH descriptor: [Leukotriene Antagonists] explode all trees

OR

montelukast OR zafirlukast OR pranlukast OR "leukotriene antagonists" OR "leukotriene receptor antagonist" OR leukotriene OR leukotriene antagonist OR Singulair OR zileuton OR verlukast OR Pobilukast OR Cinalukast

114 trial records

\section{Abbreviations}

BPD: Bronchopulmonary dysplasia; Cl: Confidence interval; IL: Interleukin; IQ: Intelligence quotient; LTRA: Antileukotriene receptor antagonist; MD: Mean difference; NICU: Neonatal intensive care unit; NRSI: Non-randomized study of intervention; RR: Risk ratio; SD: Standard deviation; GRADE: Grading of Recommendations Assessment, Development and Evaluation.

\section{Acknowledgements}

We acknowledge Cochrane neonatal for the template used in the Methods section; Maria Björklund (Library \& ICT, Faculty of Medicine, Lund University, Lund, Sweden) for designing and running the search strategy; Mari Kinoshita (Lund University, Lund, Sweden) and Yu-Tian Xiao (Department of Urology, 
Shanghai Changhai Hospital, Shanghai, China) for translating articles from Japanese and Mandarin, respectively; Prof. T. Rupprecht and Chen Xiaoqing provided additional information on their studies.

\section{Authors' contributions}

MJ screened studies for inclusion, performed data extraction and drafted the manuscript. FB screened studies for inclusion, performed data extraction and revised the manuscript. GS participated in the design of the study, screened studies for inclusion and performed data extraction. KJ provided methodological support and revised the manuscript. MB conceived of the study, participated in its design and coordination and helped to draft the manuscript. All authors read and approved the final manuscript.

\section{Funding}

Open access funding provided by Lund University.

\section{Availability of data and materials}

The data are available by accessing the published studies listed in Tables 1 and 2 .

\section{Declarations}

Ethics approval and consent to participate

Not applicable.

\section{Consent for publication}

Not applicable.

\section{Competing interests}

The authors declare that they have no competing interests.

\section{Author details}

1Amsterdam UMC, Vrije Universiteit Amsterdam, Amsterdam, The Netherlands. ${ }^{2}$ Poznan University of Medical Sciences, Poznan, Poland. ${ }^{3}$ Department of Newborns Infectious Diseases, Poznan University of Medical Sciences, Poznan, Poland. ${ }^{4}$ The Nordic Cochrane Centre, Rigshospitalet Dept., 7811, Blegdamsvej 9, 2100 Copenhagen, Denmark. ${ }^{5}$ Cochrane Sweden, Dept. Research and Development, Skåne University Hospital, Clinical Science Lund, Lund University, Lund, Sweden.

Received: 20 January 2021 Accepted: 11 July 2021

Published online: 17 July 2021

\section{References}

1. Bancalari E. Changes in the pathogenesis and prevention of chronic lung disease of prematurity. Am J Perinatol. 2001;18(1):1-10.

2. Thébaud B, Goss KN, Laughon M, Whitsett JA, Abman SH, Steinhorn RH, et al. Bronchopulmonary dysplasia. Nat Rev Dis Primer. 2019;5(1):1-23.

3. Ibrahim J, Bhandari V. The definition of bronchopulmonary dysplasia: an evolving dilemma. Pediatr Res. 2018;84(5):586-8.

4. Schmidt B, Roberts RS, Davis P, Doyle LW, Barrington KJ, Ohlsson A, et al. Caffeine therapy for apnea of prematurity. N Engl J Med. 2006;354:2112.

5. Schmidt B, Roberts RS, Davis P, Doyle LW, Barrington KJ, Ohlsson A, et al. Long-term effects of caffeine therapy for apnea of prematurity. N Engl J Med. 2007;354:1893.

6. Mastalerz L, Kumik J. Antileukotriene drugs in the treatment of asthma. Pol Arch Med Wewn. 2010;120(3):103-8.

7. Choi J, Chaudhary EA. Leukotriene receptor antagonists. 2020

8. Scott JP, Peters-Golden M. Antileukotriene agents for the treatment of lung disease. Am J Respir Crit Care Med. 2013;188(5):538-44.

9. Cardoso JO, Oliveira RV, Lu JBL, Desta Z. In vitro metabolism of montelukast by cytochrome P450s and UDP-glucuronosyltransferases. Drug Metab Dispos. 2015;43(12):1905-16.

10. Filppula AM, Laitila J, Neuvonen PJ, Backman JT. Reevaluation of the microsomal metabolism of montelukast: major contribution by CYP2C8 at clinically relevant concentrations. Drug Metab Dispos. 2011;39(5):904-11.
11. Mougey EB, Feng H, Castro M, Irvin CG, Lima JJ. Absorption of montelukast is transporter mediated: a common variant of OATP2B1 is associated with reduced plasma concentrations and poor response. Pharmacogenet Genomics. 2009;19(2):129-38.

12. Cornejo-García JA, Perkins JR, Jurado-Escobar R, García-Martín E, Agúndez JA, Viguera E, et al. Pharmacogenomics of prostaglandin and leukotriene receptors. Front Pharmacol. 2016. https://doi.org/10.3389/fphar.2016. 00316/full.

13. Haarman MG, Hunsel F, Vries TW. Adverse drug reactions of montelukast in children and adults. Pharmacol Res Perspect. 2017;5(5):e00341.

14. Aldea Perona A, García-Sáiz M, Sanz ÁE. Psychiatric disorders and montelukast in children: a disproportionality analysis of the VigiBase ${ }^{\circledR}$. Drug Saf. 2016;39(1):69-78.

15. Calapai G, Casciaro M, Miroddi M, Calapai F, Navarra M, Gangemi S. Montelukast-induced adverse drug reactions: a review of case reports in the literature. Pharmacology. 2014;94(1-2):60-70.

16. Kassahun K, Skordos K, Mclntosh I, Slaughter D, Doss GA, Baillie TA, et al Zafirlukast metabolism by cytochrome P450 3A4 produces an electrophilic alpha, beta-unsaturated iminium species that results in the selective mechanism-based inactivation of the enzyme. Chem Res Toxicol. 2005;18(9):1427-37.

17. Oñatibia-Astibia A, Martínez-Pinilla E, Franco R. The potential of methylxanthine-based therapies in pediatric respiratory tract diseases. Respir Med. 2016;1(112):1-9.

18. Resources for Review Authors. Cochrane Neonatal Rev Group [Internet] https://neonatal.cochrane.org/resources-review-authors

19. Jukema MR, Sibrecht G, Borys F, Bruschettini M. Antileukotrienes for prevention and treatment of bronchopulmonary dysplasia - systematic review of animal studies. PROSPERO 2020.

20. Jukema MR, Sibrecht G, Bruschettini M, Borys F. Antileukotrienes for the prevention and treatment of chronic lung disease in preterm newborns: a systematic review of clinical studies. PROSPERO 2020.

21. Bancalari E, Abdenour GE, Feller R, Gannon J. Bronchopulmonary dysplasia: Clinical presentation. J Pediatr. 1979;

22. Jobe AH, Bancalari E. Bronchopulmonary dysplasia. Am J Respir Crit Care Med. 2001;163(7):1723-9.

23. Walsh MC, Yao Q, Gettner P, Hale E, Collins M, Hensman A, et al. Impact of a physiologic definition on bronchopulmonary dysplasia rates. Pediatrics. 2004

24. Quinn GE. The international classification of retinopathy of prematurity revisited: an international committee for the classification of retinopathy of prematurity. Arch Ophthalmol. 2005;123(7):991-9.

25. Bayley N. Manual for the Bayley Scales of Infant Development (2nd ed.). San Antonio. 1993.

26. Bayley N. Bayley Scales of Infant and Toddler Development, Third Edition: Screening Test Manual. In: San Antonio, Texas: Pearson Clinical Assessment PsychCorp. 2006.

27. Griffiths R. The Abilities of Babies: a Study in Measurement. New York: McGraw-Hill Book Co; 1954

28. Hooijmans CR, Rovers MM, De Vries RBM, Leenaars M, Ritskes-Hoitinga M, Langendam MW. SYRCLE's risk of bias tool for animal studies. BMC Med Res Methodol. 2014;

29. Sterne JA, Hernán MA, Reeves BC, Savović J, Berkman ND, Viswanathan M, et al. ROBINS-I: A tool for assessing risk of bias in non-randomised studies of interventions. BMJ Online. 2016;

30. Sterne JAC, Savović J, Page MJ, Elbers RG, Blencowe NS, Boutron I, et al. RoB 2: A revised tool for assessing risk of bias in randomised trials. The BMJ. 2019;

31. The Cochrane Collaboration. Review Manager (RevMan). 2020.

32. Schünemann H, Brozek J, Guyatt G, Oxman A. GRADE handbook for grading the quality of evidence and the strength of recommendations [Internet]. 2013. https://gdt.gradepro.org/app/handbook/handbook.html

33. Chen YL, Cheng I, Mu SC, Yeh TF. The Clinical Impact of Leukotriene Receptor Antagonist in the Prophylaxis of. In: may 2 5; baltimore MD, united states. Baltimore MD, United States; 2009.

34. Milstein JM, Hirai C, Goetzman BW, Bennett SH. Site of action of leukotriene antagonism with FPL55712 in hypoxemic newborn lambs. Clin Res. 1988;36(1):243-243.

35. Chi CO. Research on relationship of leukotrienes and bronchopulmonary dysplasia. 2016. http://www.chictr.org.cn/showproj.aspx?proj=15045 
36. Demir K, Kumral A, Duman N, Sarioglu S, Yilmaz O, Yesilirmak DC, et al. Clarithromycin, montelukast, and pentoxifylline combination treatment ameliorates experimental neonatal hyperoxic lung injury. J Matern Fetal Neonatal Med. 2008;21(6):407-13.

37. Jouvencel P, Fayon M, Choukroun ML, Carles D, Montaudon D, Dumas E, et al. Montelukast does not protect against hyperoxia-induced inhibition of alveolarization in newborn rats. Pediatr Pulmonol. 2003;35(6):446-51.

38. Xiao Yan Y, Wang DY, Xu Y, Wang J. Effect of pranlukast on neonatal rats with periventricular leukomalacia. Chin J Contemp Pediatr. 2020

39. Chen X, Zhang X, Pan J. Effect of montelukast on bronchopulmonary dysplasia (BPD) and related mechanisms. Med Sci Monit. 2019

40. Park MS, Sohn MH, Kim KE, Park MS, Namgung R, Lee C. 5-lipoxygenaseactivating protein (FLAP) inhibitor MK-0591 prevents aberrant alveolarization in newborn mice exposed to 85\% oxygen in a dose- and timedependent manner. Lung. 2011;189(1):43-50.

41. Schreiber MD, Heymann MA, Soifer SJ. Leukotriene inhibition prevents and reverses hypoxic pulmonary vasoconstriction in newborn lambs. Pediatr Res. 1985

42. Schreiber MD, Heymann MA, Soifer SJ. The differential effects of leukotriene C4 and D4 on the pulmonary and systemic circulations in newborn lambs. Pediatr Res. 1987

43. Phillips GJ, Mohammed W, Kelly FJ. Oxygen-induced lung injury in the pre-term guinea pig: the role of leukotriene B4. Respir Med. 1995

44. Kertesz NJ, Holtzman RB, Adler L, Hageman JR. Evaluation of a leukotriene receptor antagonist in prevention of hyperoxic lung injury in newborn rabbits. Prostaglandins Leukot Essent Fatty Acids. 1992;45(2):159-65.

45. Cassin S, Gause G, Davis T, Ter Riet M, Baker R. Do inhibitors of lipoxygenase and cyclooxygenase block neonatal hypoxic pulmonary vasoconstriction? J Appl Physiol. 1989

46. Kim SB, Lee JH, Lee J, Shin SH, Eun HS, Lee SM, et al. The efficacy and safety of montelukast sodium in the prevention of bronchopulmonary dysplasia. Korean J Pediatr. 2015;58(9):347-53.

47. Rupprecht T, Rupprecht C, Harms D, Sterlacci W, Vieth M, Seybold K. Leukotriene receptor blockade as a life-saving treatment in severe Bronchopulmonary dysplasia. Respiration. 2014;88(4):285-90.
48. Kim HM, Song JE, Lee SM, Park MS, Park KI, Namgung R, et al. Montelukast as an add-on therapy in bronchopulmonary dysplasia. Korean J Pediatr. 2009;52(2):181-6

49. Panjwani D, deBoer R, Satodia P. Use of Montelukast in extreme preterm infants-a single centre experience. In: Selected Abstracts of the 6th International Congress of UENPS Session "Lung and development" [Internet]. VALENCIA (SPAIN): Journal of Pediatric and Neonatal Individualized Medicine; 2016. p. 5/19. https://jpnim.com/index.php/jpnim/article/ view/060103/400

50. Cheng I, Li-Yi T, Chen Y, Shih-Ping H, Shu-Chi M. The Use of Montelukast in Six Ventilator Dependent Infants. J Pediatr Resp Dis. 2014

51. Lui K, Lee SK, Kusuda S, Adams M, Vento M, Reichman B, et al. Trends in Outcomes for Neonates Born Very Preterm and Very Low Birth Weight in 11 High-Income Countries. J Pediatr. 2019

52. Patient Education Information Series, Treatment of Bronchopulmonary Dysplasia. Am Thorac Soc 2018;2.

53. Slater R, Moultrie F, Bax R, van den Anker J, Bhatt A. Preterm health: time to bridge the evidence gap. Lancet. 2020;396(10255):872-3.

54. Morty RE. Using experimental models to identify pathogenic pathways and putative disease management targets in bronchopulmonary dysplasia. Neonatology. 2020;117(2):233-9.

55. de Vries RBM, Wever KE, Avey MT, Stephens ML, Sena ES, Leenaars M. The usefulness of systematic reviews of animal experiments for the design of preclinical and clinical studies. ILAR J. 2014;55(3):427-37.

56. Clancy MJ. Overview of research designs. Emerg Med J. 2002;19(6):546-9.

\section{Publisher's Note}

Springer Nature remains neutral with regard to jurisdictional claims in published maps and institutional affiliations.
Ready to submit your research? Choose BMC and benefit from:

- fast, convenient online submission

- thorough peer review by experienced researchers in your field

- rapid publication on acceptance

- support for research data, including large and complex data types

- gold Open Access which fosters wider collaboration and increased citations

- maximum visibility for your research: over $100 \mathrm{M}$ website views per year

At BMC, research is always in progress.

Learn more biomedcentral.com/submissions 